NASA-TM-84396

19840008070

\title{
Special Opportunities in Helicopter Aerodynamics
}

\section{W.J. McCroskey}

\section{LIBRARY BOPY १Q⿱日一⿻口卄5




\section{Special Opportunities in Helicopter Aerodynamics}

W. J. McCroskey, Aeromechanics Laboratory, Research and Technology Laboratories U. S. Army Aviation Research and Development Command, Ames Research Center, Moffett Field, California 
SPECIAL OPPORTUNITIES IN HELICOPTER AERODYNAMICS*

W. J. McCroskey**

NASA Ames Research Center, Moffett Field, California

\author{
SUMMARY
}

Aerodynamic research relating to modern helicopters includes the study of threedimensional, unsteady, nonlinear flow fields. A selective review is made of some of the phenomenon that hamper the development of satisfactory engineering prediction techniques, but which provides a rich source of research opportunities: flow separations, compressibility effects, complex vortical wakes, and aerodynamic interference between components. Several examples of work in progress are given, including dynamic stall alleviation, the development of computational methods for transonic flow, rotor-wake predictions, and blade-vortex interactions.

\title{
I. INTRODUCTION
}

The flow fields of helicopters provide some of the most complex challenges to be found in the field of applied aerodynamics. For moderate flight conditions, a combination of practical experience, empiricism, static airfoil characteristics, and linear theory are usually adequate to estimate the overall performance and to assess the relative merits of various configuration changes. However, it is the boundaries of the flight envelope, which are typically set by vibrations, excessive power requirements, aeroelastic instabilities, and/or adverse handling characteristics, that often determine the operational success of modern helicopters. Expanding these boundaries, in turn, generally involves improving aerodynamic characteristics that are limited by very complex and nonlinear phenomena, such as boundary layer separation, and aerodynamic interference between components of the helicopter. These sundry phenomena are indicated schematically in figure 1.

This paper describes a few of the special research problems and opportunities that will be important if major improvements in helicopter characteristics are to be made in the future. The list of topics described in the following sections and in the references are only intended to be representative, and perhaps provocative, rather than complete or exhaustive. Furthermore, the material presented tends to reflect the author's personal interests, biases, background, and research activities. In each of the three general categories of viscous flows, transonic flows, and vortical wake flows, the basic aerodynamic characteristics of the particular problem are described, and one or two examples of significant research in progress are cited. A number of other important categories and research issues are mentioned in passing in the final section of the paper.

*Presented at the International Symposium on Recent Advances in Aerodynamic and Aeroacoustics, Stanford University, Aug. 1983

**Senior Staff Scientist, NASA Thermo- and Gas-Dynamics Division and U.S. Army Aeromechanics Laboratory (AVRADCOM) 


\section{ROTOR BLADE BOUNDARY LAYERS AND STALL}

The phenomenon of wing stall is well-known for fixed-wing aircraft. It limits the minimum flying speed for takeoff and landing, and hence determines the minimum runway length that is required for aircraft operations. On the other hand, rotorblade stall limits the maximum flight speed of helicopters. In blade-fixed coordinates, the difference in instantaneous local dynamic pressure on the retreating and advancing sides of the rotor disc increases monotonically with flight speed, resulting in asymmetric distributions of the sort illustrated in figure 2, which is based on the experiment of reference 1 . To maintain some semblance of rotor equilibrium, the blade-element angles of attack must be increased on the retreating blade and decreased on the advancing blade. However, there is a limit to how far the retreating blade angle of attack can be increased before the boundary layer will separate, resulting in a loss of lift and in an increase in blade-element drag and pitching moment. The variations in section lift and drag translate into limitations in rotor thrust and increases in power required, respectively; the blade torsion that is caused by the variations in pitching moment produces vibratory pitch-link loads and blade flutter.

Retreating-blade stall differs importantly in several respects from fixed-wing stall and for several different reasons, including the unsteady effects of rapidlyvarying blade-element velocity and angle of attack, and the three-dimensional effects of yaw and rotational accelerations. Experiments indicate that all of these factors may be important, but the one that seems to be predominant is the time-dependent angle of attack, or pitch rate effect. The importance of the rate of change of $\alpha(t)$ is illustrated in figure 3, which shows the lift and pitching-moment coefficient behavior for several experimental configurations. All of the unsteady cases are characterized by maximum airloads that exceed the static values and by large hysteresis in the flow behavior, according to whether the angle of attack is increasing or decreasing. The phenomenon of "dynamic stall" is characterized by strong vortical disturbances that are shed from the leading-edge region, convect over the upper surface of the airfoil, and induce highly nonlinear fluctuating airloads (ref. 2). Maximum lift is also known to be augmented by three-dimensional yaw effects (ref. 3), but the hysteresis evident in the model rotor measurements is only produced by unsteady effects.

To date, most of the research related to retreating-blade stall has been performed for the simpler model problem of a two-dimensional oscillating airfoil (fig. 3(a)), and for the associated unsteady boundary layer behavior. Furthermore, most of what is known today about the characteristics and various regimes of dynamic stall (figs. 4 and 5), has come from experiments, in two or three dimensions. Attempts to calculate the quantitative effects of dynamic stall have not been very successful up to now. Of the various two-dimensional semi-empirical correlation techniques that are available (refs. 2, 5-8), only the latest (ref. 7) has begun to demonstrate the capability of reproducing experimental results that are significantly different from the data sets that were used originally to define or tune the methods. The three-dimensional aspects of the problem remain virtually untouched.

Three principal issues have thus emerged in the general area of retreating-blade sta11. The first question is, how can the unsteady airloads be predicted with confidence over a wide range of unsteady flow conditions and blade geometries? An adequate data base already exists for the unsteady airloads on oscillating airfoils, although additional detailed measurements of boundary-layer separation characteristics would be useful. More importantly, a wide range of theoretical and numerical 
studies of unsteady viscous flows is sorely needed to provide a better basic understanding of the phenomena, to improve the predictive capability, and to assess and guide the development of more general empirical correlation techniques.

The second question is, to what extent can information derived from either theoretical or experimental studies of dynamic stall in two dimensions be applied to the real three-dimensional problem of retreating-blade stall on helicopter rotors? This issue can probably only be addressed with new special-purpose experiments, on both rotating and nonrotating three-dimensional models.

Finally, perhaps the most important issue is whether practical means can be developed to delay retreating-blade stall or alleviate its adverse consequences. Since experiments have shown even the qualitative features of dynamic stall to be surprisingly insensitive to airfoil geometry, major improvements in stall delay or control would seem to require a combination of creative new ideas for boundary-layer control or other unconventional devices, coupled with a series of careful experiments.

Figures $6-8$ show three possibilities for affecting large changes in dynamic stall characteristics, by pneumatic or mechanical means. Although these sorts of devices have been used successfully in fixed-wing applications, only circulation control airfoils have been incorporated into an experimental helicopter rotor (ref. 9). However, much remains to be learned about the performance of either leading-edge or trailing-edge blowing under unsteady, three-dimensional, and separated flow conditions. The situation is likely to be further complicated by the development of transonic flow near the leading edge (see Section III), if the blowing delays the onset of dynamic stall to significantly higher values of lift coefficient; that is, if it is successful.

With regard to mechanical devices, Carr and McAlister (ref. 11) have found the leading-edge slat configuration shown in figure 8 to be effective in suppressing dynamic stall on an airfoil undergoing sinusoidal oscillations in pitch, as shown in figure 9. Their particular test configuration of a fixed-geometry slat suffered from high values of $C_{D}$ at low angles of attack, but the dramatic improvement in $C_{L}$ and $\mathrm{C}_{\mathrm{M}}$ indicates that the concept is worthy of further exploration and refinement. As in the blowing cases, three-dimensional and transonic effects will also have to be studied before we can expect this device to be incorporated into an actual rotor. In any case, this or any other boundary-layer control device that could enable a rotor blade to approach the high-lift capabilities of modern airplane wings would pay handsome dividends for helicopter rotors.

\section{TRANSONIC AERODYNAMICS}

Transonic flow phenomena commonly appear on the advancing blade tips of most modern helicopter rotors, where the blade-element Mach numbers are high; for example, as shown in figure 2(a). It should be emphasized that shock waves may also appear on the retreating blade as well, when the blade-element angle of attack is sufficiently high to produce local regions of supersonic flow near the leading edge. Thus the transonic airfoil problem for rotors includes both of the facets illustrated in figure 10. The periodic development and decay of shock waves on rotor blades in forward flight lead to vibratory airloads, degradations in performance and aerodynamic efficiency, and excessive noise. 
In addition to the unsteady complications, the transonic flow on the advancing blade is highly three-dimensional, generally rendering two-dimensional information much less valuable than for the retreating-blade stall discussed above. Figure 11 shows representative spanwise variations in the pressure coefficient at the quarterchord position on the advancing blade, along with an indication of the various flow regimes that develop. The change in the chordwise pressure distribution along the span is also indicated. In the figure, the transonic similarity parameter, following Isom (ref. 12), is the following:

$$
K=\frac{1-M_{T}^{2}(r+\mu \sin \psi)^{2}}{\left[(\gamma+1) M_{T}^{2}(r+\mu \sin \psi)^{2} \tau\right]^{2 / 3}}
$$

where $\mathrm{M}_{\mathrm{T}}$ is the tip Mach number in hover, $\psi=\Omega \mathrm{t}$ is the azimuthal position of the blade, $r$ is the nondimensional radial dimension, $\mu=V_{\infty} / \Omega R$ is the advance ratio, $\tau$ is the airfoil thickness ratio, and $\gamma$ is the ratio of specific heats. The inverse of $\mathrm{K}$ essentially represents the order of magnitude of the most important nonlinear term in the governing flow equation relative to the size of the linear terms. In practice, the relative importance of the various regions, especially Region 2 vs Region 4 vs Region 5, depends upon the flight conditions and the blade geometry. In all cases, however, the flow in the outboard region's is threedimensional, and calculations also show important unsteady effects to occur in forward flight, when the flow there is transonic.

It is important to recognize three essential differences between the transonic aerodynamics of fixed-wing aircraft and helicopter blades. The first is that the blade-element Mach number increases linearly with distance from the axis of rotation. This enhances transonic effects near the blade tip, in opposition to and in competition with the three-dimensional tip-relief effect there. This tip relief, of course, is basically the same as for a nonrotating wing. However, the behavior beyond the tip is different, because of the linearly-varying Mach number; see reference 13.

The second important difference is that in forward flight the blade-element Mach number and angle of attack vary with time, or rotor blade azimuthal position, as shown in figure 2. This effect is relatively unimportant in the inboard, linear regimes, but the outboard transonic flow field takes longer to develop, and unsteady effects become essential features of the flow at high advance ratio. Because of the lag in adjusting to the time- and space-varying local Mach numbers, the flow in the second quadrant, $90^{\circ}<\psi<180^{\circ}$, generally has stronger and more persistent shock waves than that of the first quadrant, $0<\psi<90^{\circ}$.

The third difference, also of great importance, is the presence of concentrated tip vortices that trail from the tips of preceding blades. Depending on the flight conditions, these trailing vortices may remain near the path of the advancing blade, causing large disturbances to the shock-wave development and decay. This aspect of the problem cannot be uncoupled from the rotor wake structure, described in Section IV, making it probably the most difficult and challenging aspect of the aerodynamics of the advancing blade.

Still another factor that is becoming increasingly important is the trend toward exotic tip geometries for advanced rotor designs. Figure 12, adapted from reference 14, illustrates some examples which are already used on modern helicopters or are being developed. These various planforms are designed to alleviate shock-wave development by sweep effects, to reduce vibratory loads by either aerodynamic or 
structural dynamic changes, to increase hover performance by changes in spanwise circulation distribution, or to combine several of these factors. The systematic evaluation of such tip shapes is a difficult task beyond our present capabilities. In addition, each of the planforms in figure 12 would require some adaptation of the computational grid in any numerical analysis of the tip region. Thus, the subject of grid generation has become yet another fertile area for research.

The accurate treatment of the transonic regimes on rotor blades is, therefore, a formidable challenge. Fortunately, considerable assistance can be obtained from the large background of computational aerodynamics methods that have been developed over the past decade for fixed-wing applications, despite the aforementioned differences. Although it is by no means a trivial exercise to include both unsteady and threedimensional effects, existing solution algorithms for the transonic small-disturbance, full potential, Euler, and even the Navier-Stokes equations, with sufficient effort, could be adapted to helicopter problems. Significant progress has already been made in recent years; and the level of complexity that has been implemented includes the following:

(1) The unsteady, three-dimensional transonic sma11-disturbance equation for a lifting blade with an approximate wake model, e.g., figure 13 from reference 15 .

(2) The quasi-steady full-potential equation for a highly-swept, nonlifting blade, e.g., figure 14 from reference 16 .

(3) The unsteady full-potential equation for a nonlifting blade, e.g., figure 15 from unpublished work by I. C. Chang at NASA Ames Research Center.

It should be mentioned that for realistic rotor calculations, the transonic fiəld calculations have to be coupled with the rotor wake, which is discussed in more detail in Section IV. At present it is not practical to do more than solve the governing equations by finite-difference methods on an isolated blade and within a limited computational volume around the blade tip. Therefore, the information about the vortex system in the wake and the associated rotor-induced inflow is accounted for either as prescribed modifications to the boundary conditions on the outer boundary of the computational box, or as an additional interior-flow boundary condition that is prescribed within the computational box, or both. This is indicated schematically in figures 16 and 17. As shown in figure 17, the interior of the computational domain includes the usual trailing sheet of vorticity behind the blade, which is assumed to be undistorted, and one or more segments of line vortices representing the trailing tip vortex (or vortices) from the preceding blade(s). The line vortex segments are connected with the outer boundary by branch-cut surfaces, across which jumps in potential are imposed that correspond to the strengths of the concentrated vortices.

The foregoing procedure makes it possible to perform meaningful transonic calculations for special cases. However, further improvements are required in two rather diverse directions. First, the existing codes are still too expensive and complex, in general, for routine use in engineering design. Therefore, serious efforts are needed to streamline the methodology and to improve the computational efficiency, without losing the essential features of the problem. On the other hand, the current assumptions and restrictions are still too severe, in general, to allow for accurate simulations of many of the most crucial operational conditions of advanced rotor systems. Therefore, improved transonic aerodynamics modeling is needed, but it must 
not add excessive burdens to the computational requirements. Clearly this area offers considerable opportunities and challenges for future research.

\author{
IV. ROTOR WAKES AND VORTICES
}

\title{
A. Global Wake Modeling
}

Accurate prediction of the vortical wake of a helicopter rotor is probably the most important, the most studied, and the most difficult aspect of helicopter aerodynamics; consequently, it can be argued that this topic provides one of the greatest opportunities for new improvements. Certainly the treatment of the wake is crucial in terms of performance, efficiency, structural vibrations, and aerodynamic noise. Current methods of analysis range from relatively simple momentum-theory applications to free-wake lifting-surface codes that consist of several thousand lines of Fortran statements and that require approximately one million words of computer memory. In between these two extremes, there are a variety of so-called prescribed-wake models, which rely on some degree of empiricism to determine the position of the wake vortices; then the Biot-Savart law is used to calculate the velocity field that is induced by these vortices at the plane of the rotor blades.

The complexity of the problem in comparison with fixed-wing aircraft is illustrated in figures 18 and 19. The detailed structure of an airplane wake is not all that simple, but because the wake elements trail rearward along approximately straight lines that are parallel to the flight direction, simple approximations to the tipvortex rollup and downstream convection normally suffice. This is not at all the case for the helicopter rotor, whose blade tips trace out prolate cycloidal paths in space. This provides numerous opportunities for complex interactions between the vortices and the blades and between the vortices themselves.

It is beyond the scope and intent of this paper to review the state of the art in rotor wake modeling; a few examples are given to point out some features of the problem and to mention some of the limitations of existing methods. For example, figure 19 from reference 17 is representative of the latest generation of prescribed wake analyses; this is an extension to forward flight of one of the better-known generalized prescribed-wake methods for hover (ref. 18). This method is simpler and less expensive than the free-wake codes of comparable accuracy.

However, an inherent limitation of the prescribed-wake approach is that the empirical determination of the wake shape ignores some of the details of the particular case under consideration, including the mutual interaction between vortex elements. As a result, a prescribed-wake configuration is not, in general, a valid solution to an inviscid free-vortex flow (ref. 19). In most cases where the wake geometry is not too different from the configurations that produced the original empiricism, this may not be a serious error. However, prescribed-wake models are unreliable for unusual blade planforms and/or twist distributions, and these are often the cases that are the most interesting to explore.

In some of these more challenging cases, the blade airloads depend strongly upon both the wake geometry and the method for calculating the flow. A relatively simple example of this is given in figure 20. This figure, prepared by $\mathrm{Dr}$. Chee Tung of the U.S. Army Aeromechanics Laboratory, shows a comparison of lifting-line and liftingsurface calculations with the hover data of Caradonna and Tung (ref. 20) for a mode1 rotor with two low-aspect-ratio blades of rectangular planform and zero twist. The 
computed results were obtained using the computer code described in reference 21 . Here the local lift coefficient is nondimensionalized by the tip speed, $\Omega$.

For this example, the lifting-line calculations were in error, regardless of the wake mode1. The prescribed-wake option of the program also gave an erroneous spanwise load distribution, regardless of which representation was used for the surface of the blade, primarily because the prescribed wake geometry was not correct. On the other hand, the free-wake calculation predicted the actual wake geometry reasonably we11. Consequently, the lifting-surface calculation gave essentially the same satisfactory results either when using the wake prescribed according to the experimental measurements or when using the free-wake option of the code. As a final footnote, however, Dr. Tung reports that this same free-wake, lifting-surface code fails to predict adequately some cases with highly nonlinear twist distributions. This exercise illustrates the difficulty and importance of the wake geometry for the problem of a rotor in hover, and it indicates that further work is needed even without the complications of forward flight.

\section{B. Blade-Vortex Interaction}

The strong interaction between a segment of a rotor blade and the concentrated tip vortices in the wake is an important potential source of noise and vibration at low and moderate flight speed. The generic problem, sketched in figure 21 , is an unsteady, three-dimensional close encounter of a curved line vortex, at an arbitrary intersection angle, $\Lambda_{i}$, with a high-aspect-ratio lifting surface that is executing combined rotational and translational motion at transonic speeds. For many practical applications, it is almost impossible to separate the transonic aspects of the problem from the details of the vortex structure in the wake.

Insofar as basic research opportunities are concerned, the limiting cases of $\Lambda_{i}=0$ and $90^{\circ}$, as shown in figure 22, are of particular interest. The former case is fundamentally unsteady but approximately two-dimensional, whereas the latter is essentially steady but highly three-dimensional. These two limiting cases are the basis of several ongoing research programs that are designed to explore the basic features of blade-vortex interactions, to develop methods of calculating such interactions, and to determine the minimum level of complexity that will be required in the future for adequate predictive capability in the complete rotor environment. The case of a vortex that is parallel to the free stream has received more attention in the past, as it is more relevant to fixed-wing applications and it is relatively easy to set up experimentally. On the other hand, the case of the vortex that is parallel to the leading edge is a simpler problem for theoretical and numerical analysis, and it requires less computer memory to store the solution variables.

For illustrative purposes, we shall briefly review some recent numerical work for the two-dimensional unsteady case, $\Lambda_{i}=0$. Two of the basic issues are the introduction of the vortex into the numerical computations and the determination of its effect on the unsteady pressure distribution on the airfoil and nearby. The vortex can be introduced in two different ways, as indicated in figure 23. Caradonna, et al (ref. 15) and George and Chang (ref. 22) have utilized the branch-cut method, as described in Section III, and McCroskey and Goorjian (ref. 23) employed the prescribed-disturbance method. A11 three groups solved the transonic smalldisturbance equations by time-accurate ADI methods that had been developed and checked out previously, and the various results are in reasonable agreement in most cases. 
Representative results from reference 23 are shown in figures $24-25$ for two symmetrical airfoil sections. Figure 24 shows the distortions in the chordwise pressure distributions on the airfoil as the vortex passes underneath. Similar results are shown in figure 25 for a thinner airfoil, including the time histories at individual locations on the airfoil. These results illustrate that strong gradients in pressure occur with respect to both time and space, due to vortex encounter. These gradients can be especially significant in the leading-edge region of a thin airfoil.

Although the subject of airfoil-vortex interaction is just beginning to receive significant attention, several important features of the problem have already emerged. First, the effect of the vortex is felt primarily through the vertical velocity that it induces, which to first order appears as a time-dependent perturbation in the effective angle of attack, and, secondarily, through its horizontal induced velocity. Second, unsteady lag effects are very important, especially in the transonic case; calculations show enormous differences between quasi-steady and unsteady solutions and between the results for the vortex locations upstream and downstream of the airfoil. Third, other strong nonlinear effects have been noted; even the qualitative pressure variations with respect to time and space differ markedly from the predictions of linear theory, and the vortex distorts the flow on the nearest surface of the body much more than on the opposite surface. Finally, some of the pressure perturbations appear to leave the body as radiating waves; this phenomenon would seem to represent the far-field blade-vortex interaction noise of helicopter rotors.

The studies to date (refs. 15, 22-23) have been performed with the smalldisturbance equations, whose accuracy is questionable in the very region that seems to be the most important (ref. 13); that is, near the leading edge. Therefore, it seems imperative to check the small-disturbance codes with more accurate formulations and with experiments. Also, an ideal potential vortex with an invariant structure has been assumed thus far; but in reality, close encounters probably alter the vortex core significantly and may lead to vortex bursting. For rotor applications, future studies must be extended to include three-dimensions, where it will be even more important to establish what minimum level of complexity in the governing equations will suffice and to determine the most expeditious way to introducing the vortex into the computational domain. Finally, the severity of the blade-vortex interaction appears to be highly sensitive to the strength and position of the vortex, especially for transonic flow; therefore, the results of the computations will be no better than the predictions of the wake model used.

\section{CONCLUDING REMARKS}

The examples discussed in the preceding sections are but a few of the myriad of serious aerodynamic problems of helicopters that provide rich opportunities for future research. There are other examples which are not discussed in this selective review; however, there are several that are worth mentioning in passing.

The first is the general category of aerodynamic interference. As indicated in figure 1 , the flow field of the various components of the helicopter frequently influence other flow regions, and these interactions can be complex and nonlinear. Typical examples include the mutual interference and interaction between the main rotor and the fuselage, between the main rotor and the tail rotor, between the rotor hub and the fuselage, and between the tail rotor and the tail surfaces. Panel methods are being developed for predicting these flows; e.g., reference 24 , but the current industry approach is mostly empirical. A closely-related problem that has 
arisen recently is the rotor-induced "download" on the wing of the tilt-rotor aircraft, figure 26. In hover, the downwash from the rotors impinges on the wing, causing a net loss in lifting capability of $10 \%$ or more of the gross weight of the aircraft. Predicting this effect accurately and designing flap configurations to minimize the download are particularly challenging problems, but ones which have great payoff.

Another important area is that of simply improving the aerodynamic tools that are available to helicopter engineers. The need for simpler and more efficient computational techniques that can more accurately solve more complex problems has already been stressed. However, improvements in the coupling of aerodynamic prediction techniques with structural-dynamics codes is also essential. In addition, there would appear to be tremendous opportunities for coupling efficient aerodynamic codes with numerical optimization techniques to design improved blade configurations. This has already been demonstrated in two dimensions for transonic rotor airfoil sections (ref. 25). The extension to three dimensions, that is, including planform and twist distributions, is the next logical step, although it is obviously a very large step.

Experiments will continue to play a major role in helicopter technology for the foreseeable future, and there is much to be done in the area of instrumentation, experimental methods, and testing techniques. One perennial issue is the gap between model and full-scale testing; the recent paper by Keys, et al (ref. 26), for example, discusses some aspects of this problem. Mach number scaling is clearly essential; but this is hardly a research issue any more, even though constructing dynamicallyscaled, instrumented model rotors to operate at full-scale tip speeds can be a vexing engineering exercise, On the other hand, typical Reynolds numbers for model and fullscale helicopters span regimes where the boundary-layer transition and separation characteristics may be considerably different. Therefore, the model/full-scale issue will continue to be important for any aspect of helicopter aerodynamics in which viscous effects play a significant role.

A strong need for a wide variety of special-purpose experiments will continue to exist. For example, tests of complete rotor systems certainly have their place, but a full-scale helicopter blade is not the best experimental configuration to study the effects of unsteadiness on the Reynolds stresses of a separating turbulent boundary layer, nor to validate initially a new transonic code. Relatively simple experiments that are run under we1l-controlled and thoroughly-documented conditions are essential to sort out the dominant features of the various flow fields, to increase our fundamental understanding of the basic phenomena, to guide the development of new theoretical models, and to validate methodically the new computational algorithms and prediction techniques as they are being developed.

Finally, for both the global studies and for the special-purpose experiments, a wide variety of new instrumentation, measurement techniques, and data acquisition and processing methods need to be developed. These are required to cope with the complexity of the unsteady three-dimensional flow fields of the various helicopter components and with handling the large volume of data that are generated. It is as challenging to measure the right information and to eliminate the superfluous, as it is to develop the new predictive methodology.

In conclusion, the examples discussed in this paper illustrate the complexity of helicopter aerodynamics and the many important issues that remain to be studied. There are numerous challenges and a wide range of opportunities for the technologist 
and for the basic research scientist to explore. Their various contributions will assure continuing major improvements to this unique flying machine.

\section{REFERENCES}

1. Rabbott, J. P., Jr.; Lizak, A. A.; and Paglino, V. M.: A Presentation of Meassured and Full-Scale Rotor Blade Aerodynamic and Structural Loads. US Army AVLABS Tech. Report 66-31, 1966.

2. McCroskey, W. J.: The Phenomenon of Dynamic Stal1. NASA TM 81264, 1981.

3. St. Hilaire, A. O.; Carta, F. O.; and Fink, M. R.: The Influence of Sweep on the Aerodynamic Loading of an Oscillating NACA 0012 Airfoil. NASA CR-3092, 1979.

4. McCroskey, W. J.; and Fisher, R. K.: Detailed Aerodynamic Measurements on a Model Rotor in the Blade Stall Regime. J. Am. Hel. Soc., vol. 17, no. 1, Jan. 1972, pp. 20-30.

5. Harris, F. D.; and Pruyn, R. R.: Blade Stall - Half Fact, Half Fiction. J. Am. Hel. Soc., vol. 13, no. 2, Apr. 1968, pp. 27-48.

6. Tran, C. T.; Petot, D.; and Falchero, D.: Aeroelasticity of Helicopter Rotors in Forward Flight. la Recherche Aérospatiale, vol. 1982, no. 4, JuilletAôut, 1982, pp. 11-25.

7. Gangwani, S. T.: Synthesized Airfoil Data Method for Prediction of Dynamic Stall and Unsteady Airloads. Proc. Am. He1. Soc. Forum, Paper No. A-83-39-0020000 , May 1983.

8. Ericsson, L. E.; and Reding, J. P.: The Difference Between the Effects of Pitch and Plunge on Dynamic Airfoil Stall. Proc. Ninth European Rotorcraft Forum, Paper 8, Sept. 1983.

9. Williams, R. M.: Application of Circulation Control Rotor Technology to a Stopped Rotor Aircraft Design. Vertica, vol. 1, no. 1, July 1976, pp. 3-15.

10. McCloud, J. L., III; Ha11, L. P.; and Brady, J. A.: Ful1-Scale Wind -Tunnel Tests of Blowing Boundary-Layer Control Applied to a Helicopter Rotor. NASA TN D-335, 1960.

11. Carr, L. W.; and McAlister, K. W.: The Effect of a Leading-Edge Slat on the Dynamic Stall on an Oscillating Airfoil. AIAA Paper 83-2533, Oct. 1983.

12. Isom, M. P.: Unsteady Subsonic and Transonic Potential Flow Over Helicopter Rotor Blades. NASA CR-2463, Oct. 1974.

13. Schmitz, F. H.; and Yu, Y. H.: Helicopter Impulsive Noise: Theoretical and Experimental Comparisons. Recent Advances in Aerodynamics and Acoustics, Stanford University, Aug. 1983, and NASA TM 84390 in press.

14. Philippe, J. J.; and Vuillet, A.: Aerodynamic Design of Advanced Rotors With New Tip Shapes. Proc. Am. Hel. Soc. Forum, Paper A-83-39-04-0000, May 1983. 
15. Caradonna, F. X.; Desopper, A.; and Tung, C.: Finite Difference Modeling of Rotor Flows Including Wake Effects: NASA TM-84280, Aug. 1982.

16. Tauber, M. E.; Chang, I-C.; Caughey, D. A.; and Philippe, J. J.: Comparison of Calculated and Measured Pressures on Straight and Swept-Tip Model Rotor Blades. NASA TM-85872 in preparation.

17. Egolf, T. A.; and Landgrebe, A. J.: Helicopter Rotor Wake Geometry and Its Influence in Forward Flight. NASA CR-3726, 1983.

18. Landgrebe, A. J.; Moffitt, R. C.,; and Clark, D. R.: Aerodynamic Technology for Advanced Rotorcraft. J. Am. Hel. Soc., vol. 22, no. 2, Apr. 1977, pp. 21-27.

19. Bliss, D. B.; Quackenbush, T. R.; and Bilanin, A. J.: A New Methodology for Helicopter Free Wake Analysis. Proc. Am. Hel. Soc. Forum, Paper A-83-39-750000 , May 1983.

20. Caradonna, F. X.; and Tung, C.: Experimental and Analytical Studies of a Model Helicopter Rotor in Hover. NASA TM-81232, Sept. 1981.

21. Summa, J. M.: Advanced Rotor Analysis Methods for the Aerodynamics of Vortex/ Blade Interactions in Hover. Proc. Eighth European Rotorcraft Forum, Paper 2.8, Sept. 1982 .

22. George, A. R.; and Chang, S. B.: Noise Due to Transonic Blade-Vortex Interactions. Proc. Am. Hel. Soc. Forum, Paper A-83-39-50-0000, May 1983.

23. McCroskey, W. J.; and Goorjian, P. M.: Interactions of Airfoils With Gusts and Concentrated Vortices in Unsteady Transonic F1ow. AIAA Paper 83-1691, July 1983.

24. Clark, D. R.; and Maskew, B.: Aerodynamic Modeling of Helicopter and Tilt Rotor Configurations. Proc. Am. Hel. Soc. Forum, Paper A-83-39-72-0000, May 1983.

25. Hicks, R. M.; and McCroskey, W. J.: An Experimental Evaluation of a Helicopter Rotor Section Designed by Numerical Optimization. NASA TM-78622, Mar. 1980.

26. Keys, C. N.; McVeigh, M. A.; Dadone, L.; and McHugh, F. J.: Considerations in the Estimation of Full-Scale Rotor Performance From Model Rotor Test Data. Proc. Am. Hel. Soc. Forum, Paper A-83-39-03-0000, May 1983. 


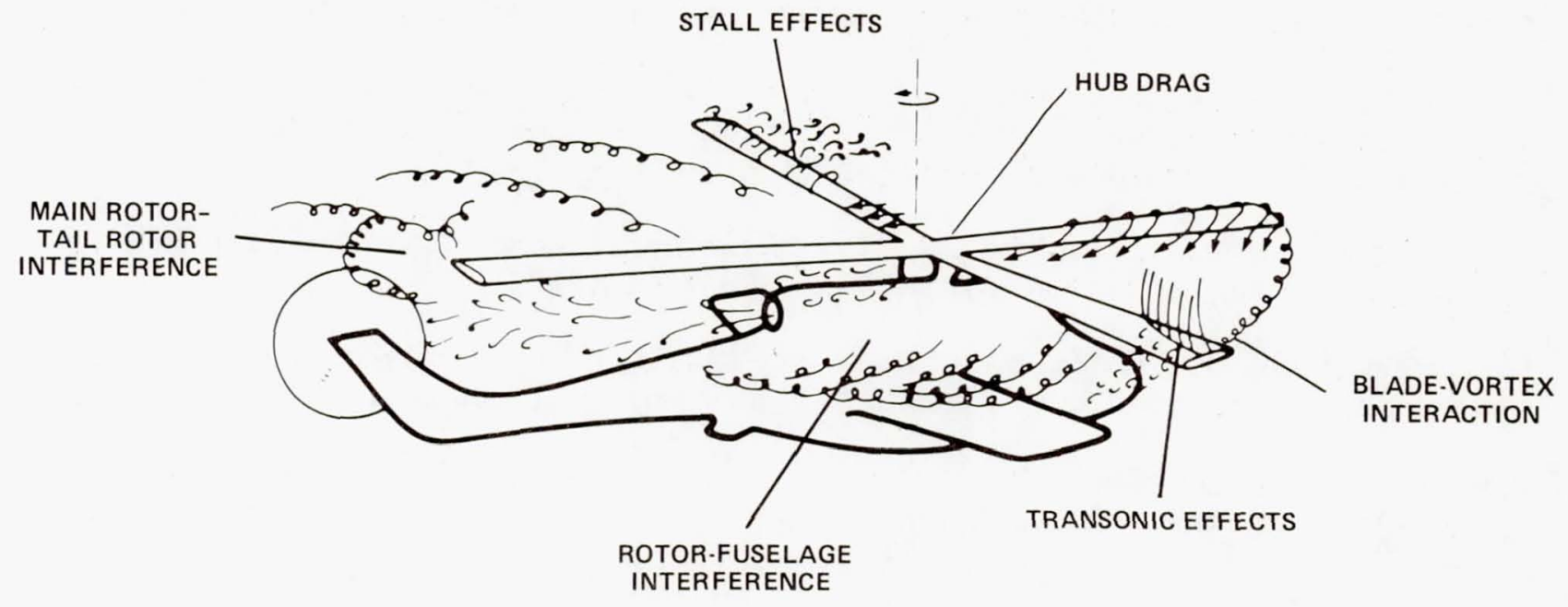

Figure 1.- Major problem areas in helicopter aerodynamics.
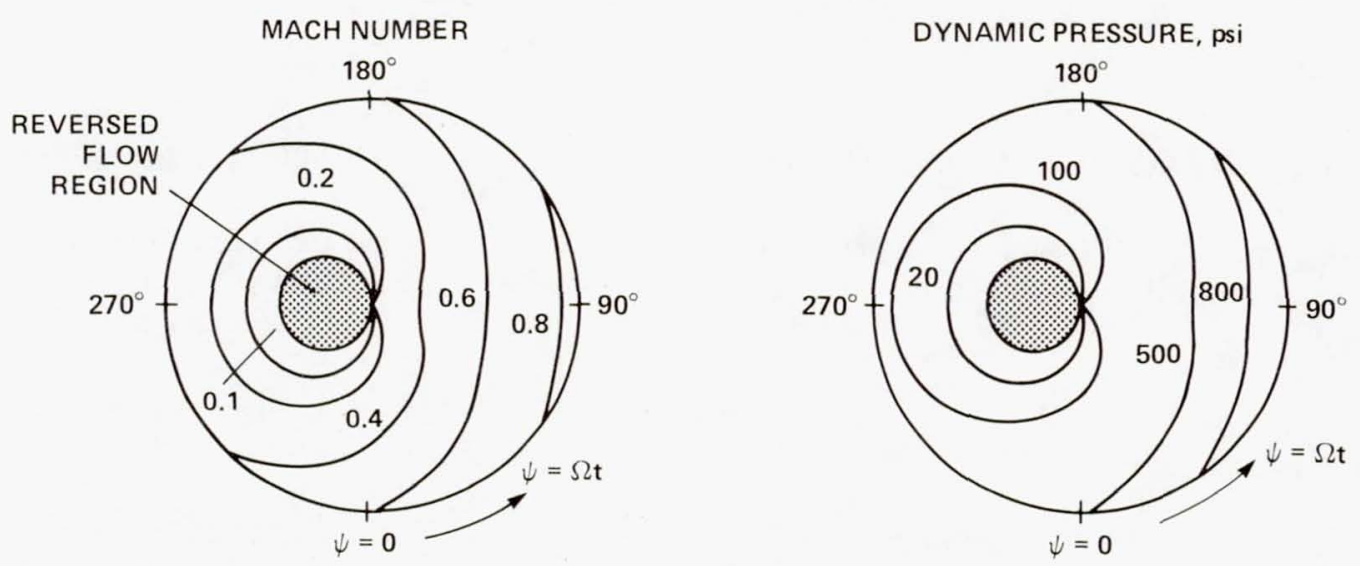

ANGLE OF ATTACK, deg
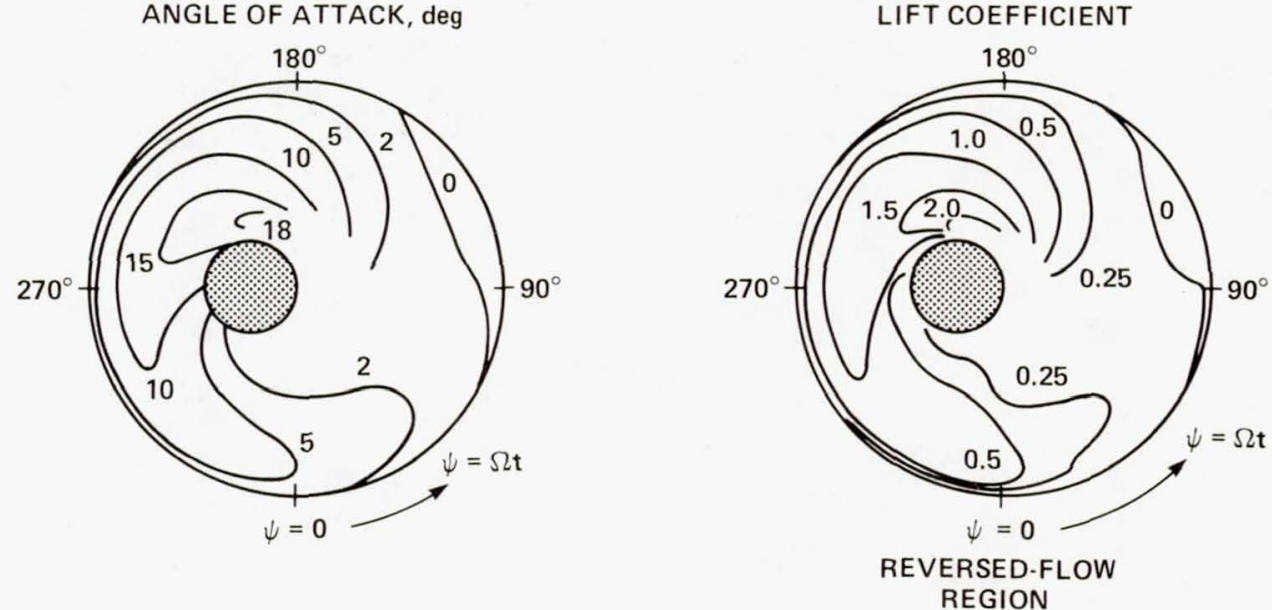

Figure 2.- Blade-element conditions for a lightly-loaded rotor in high speed forward flight, $V_{\infty}=175$ knots (ref. 1$) ; \mu=0.45, C_{\mathrm{T}} / \sigma 0.05$. 

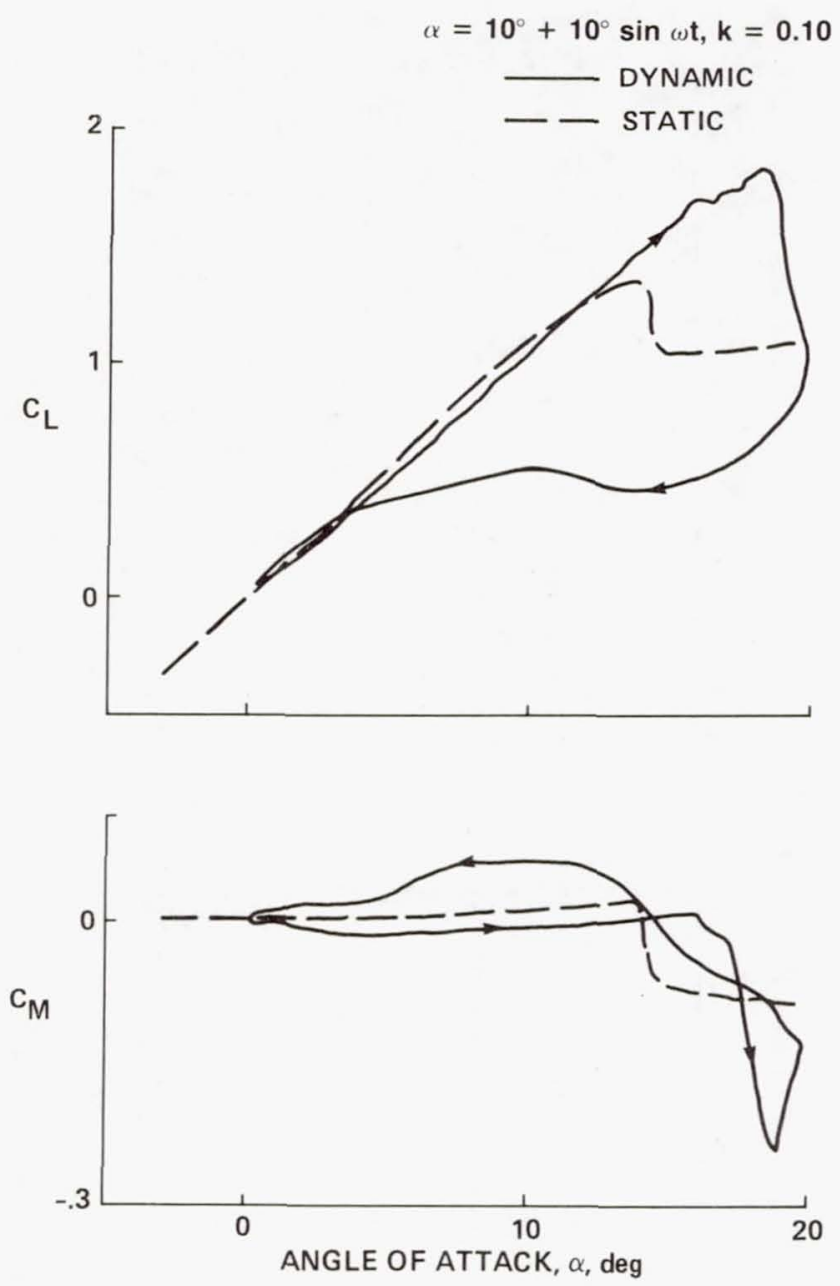

(a) Unsteady two-dimensional flow, pitching oscillations (ref. 2).

Figure 3.- Lift and pitching-moment behavior vs. aerodynamic angle of attack. 

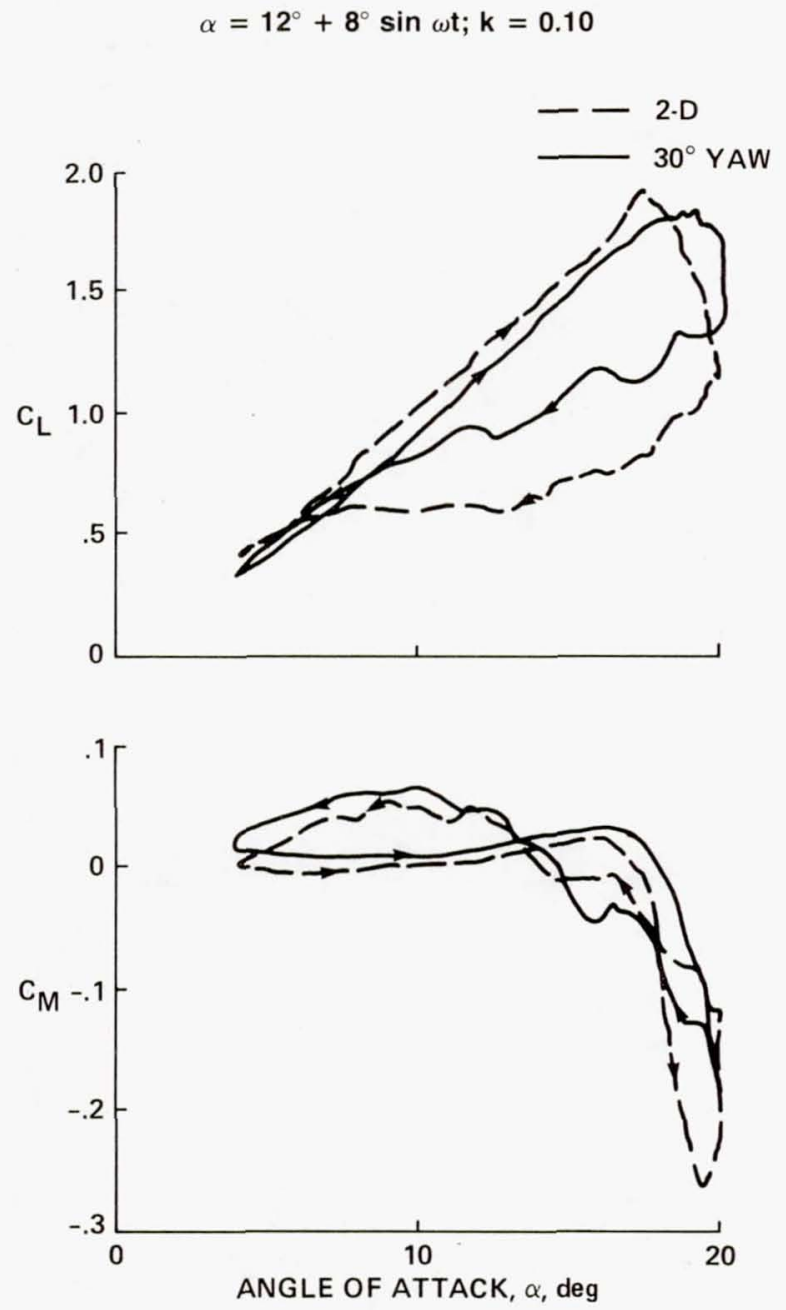

(b) Unsteady yawed flow, pitching oscillations (ref. 3).

Figure 3.- Continued. 
$\mu=0.35, r / R=0.75$

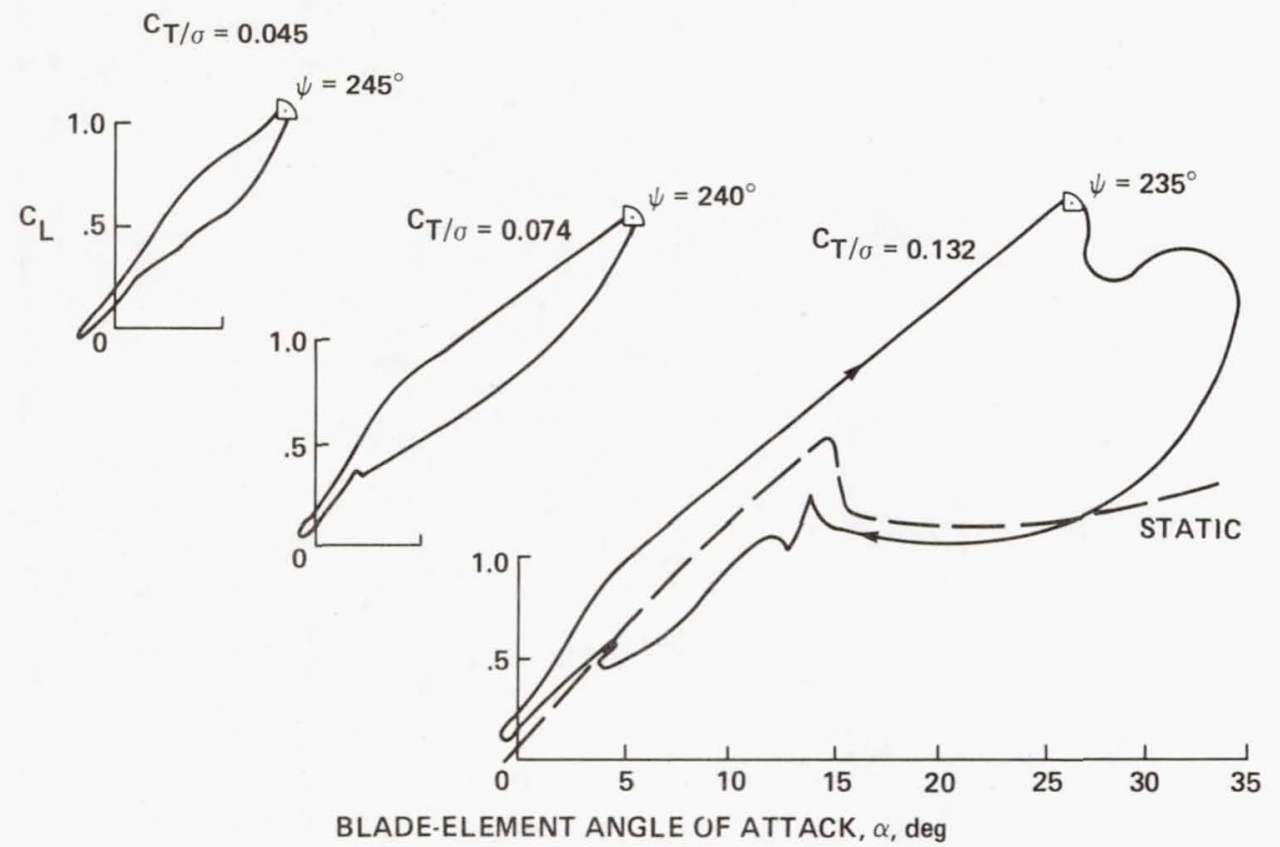

(c) Model rotor blade in forward flight (ref. 4).

Figure 3.- Concluded. 
$\alpha=5^{\circ}+10^{\circ} \sin \omega t, \quad k=0.1$
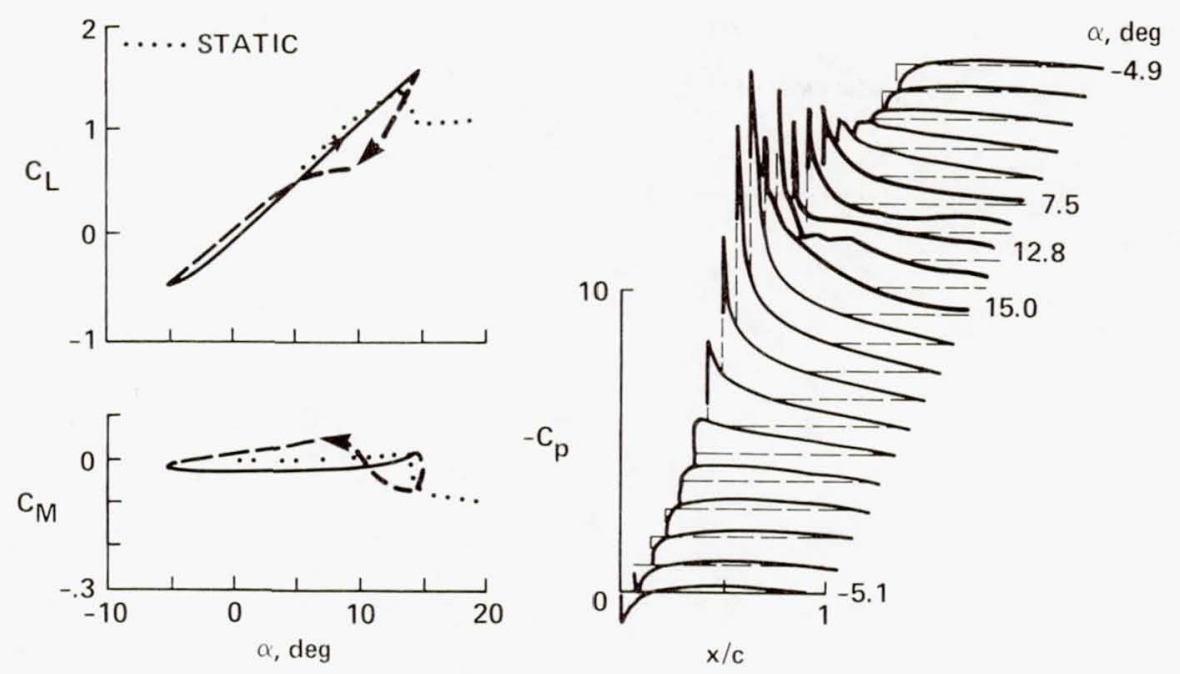

TRAILING-EDGE SEPARATION

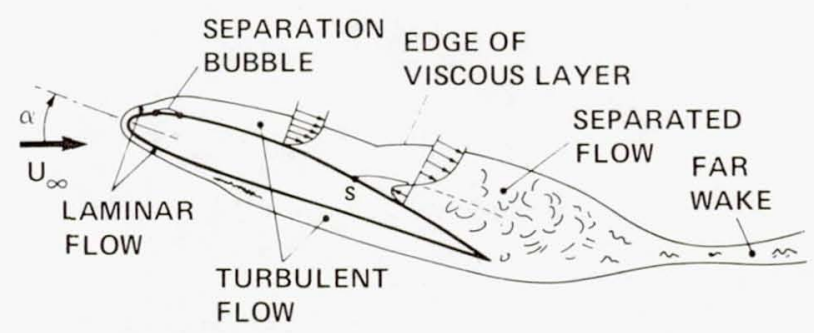

- STRONG INTERACTION

- VISCOUS LAYER = (AIRFOIL THICKNESS)

Figure 4.- Light dynamic stall on an oscillating airfoil. 

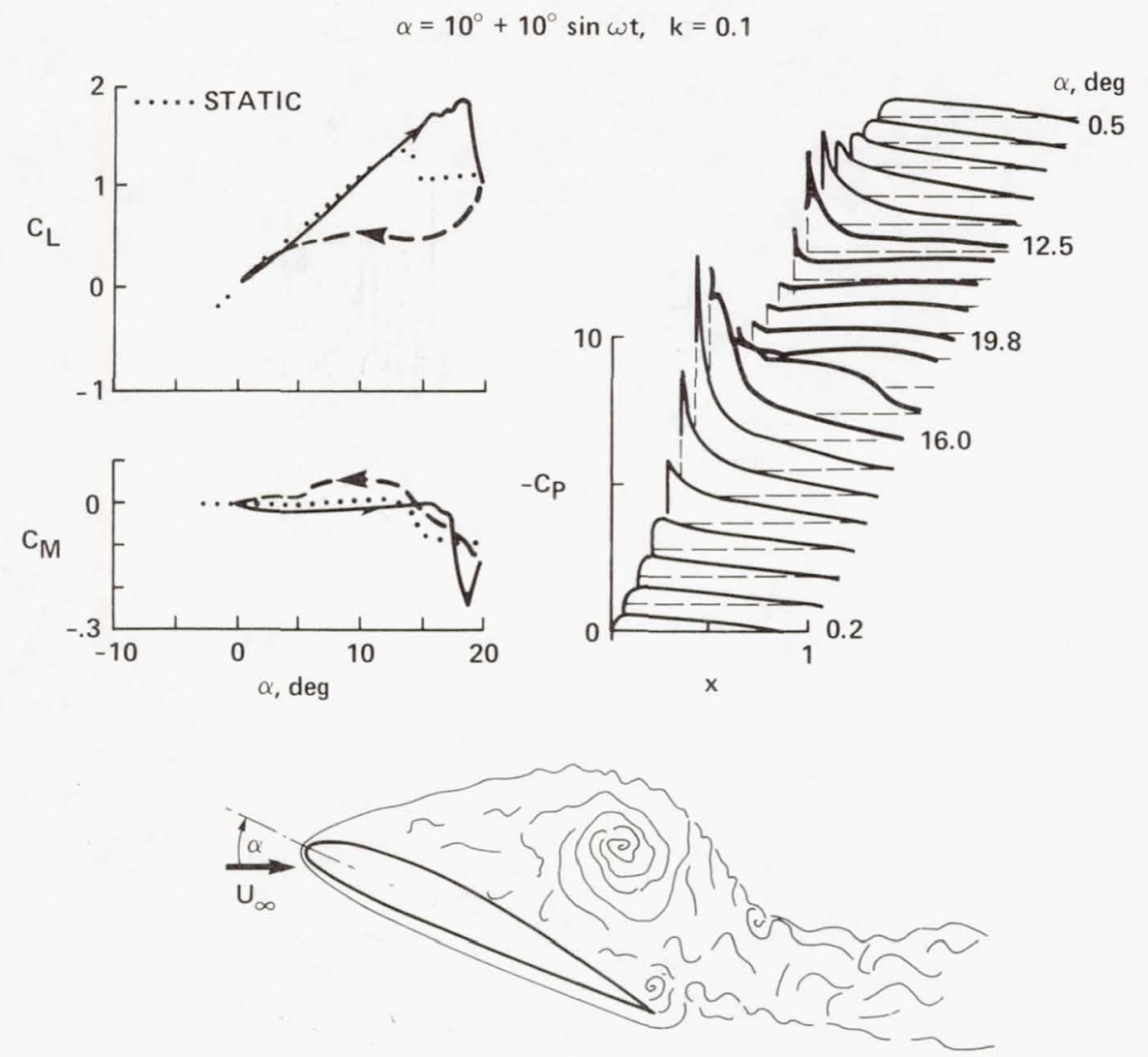

- VORTEX DOMINATED

- VISCOUS LAYER = $O$ (AIRFOIL CHORD)

Figure 5.- Deep dynamic stall on an oscillating airfoil. 

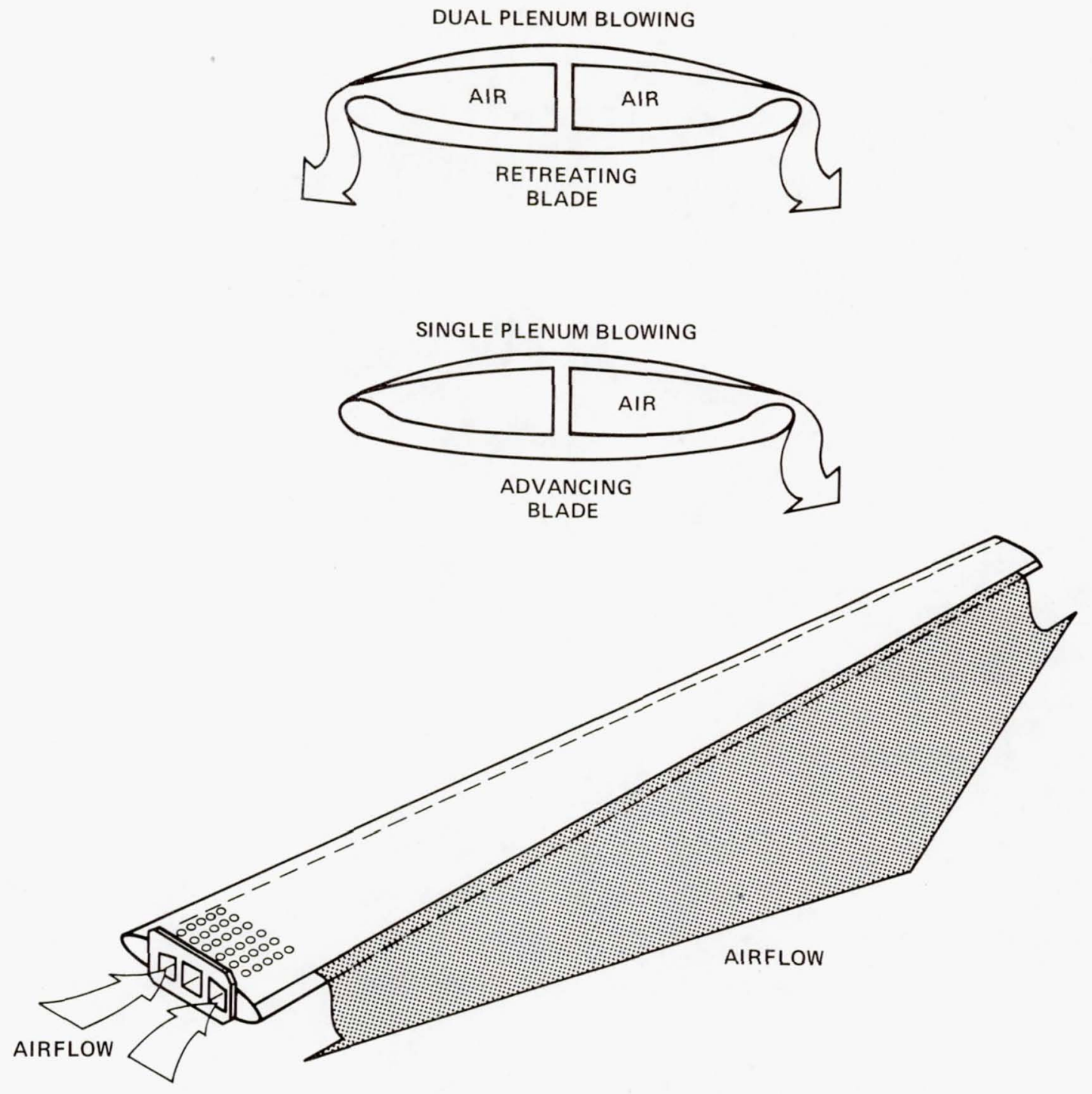

Figure 6.- Circulation control to alleviate retreating blade stall (ref. 9). 


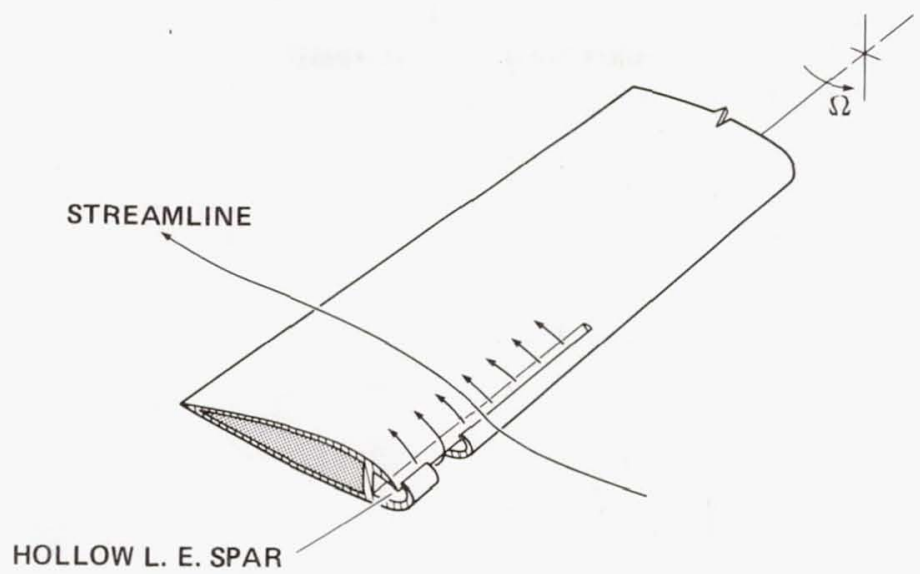

Figure 7.- Leading-edge blowing to alleviate retreating blade stall (ref. 10).
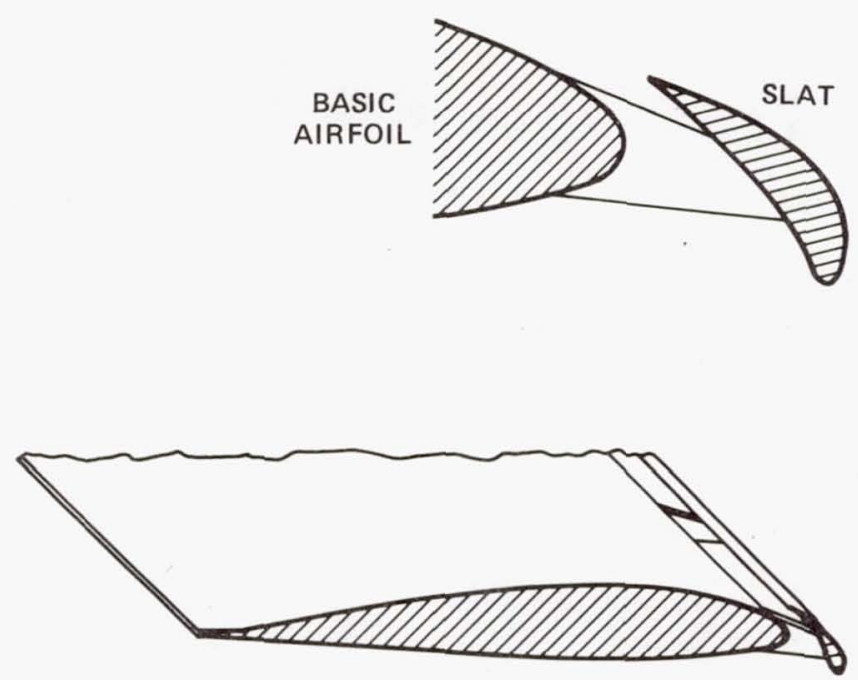

Figure 8.- Leading-edge slat to alleviate retreating blade stall (ref. 11). 

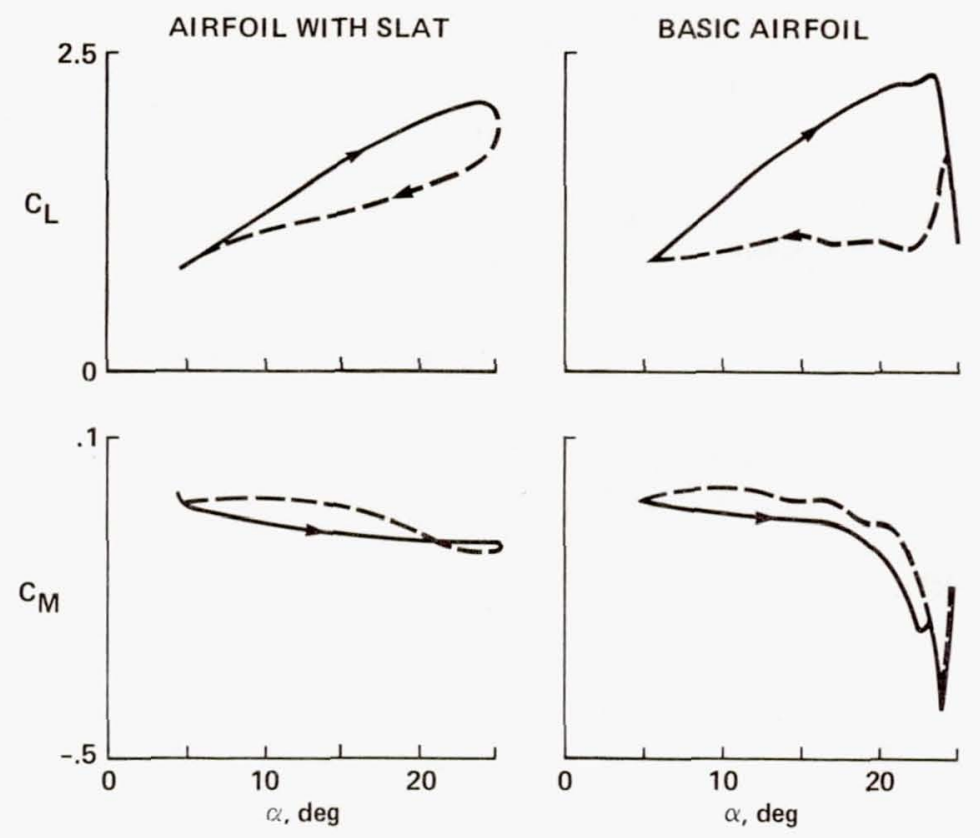

Figure 9.- Comparison of lift, moment, and pressure distributions for a helicopter airfoil with and without a leading-edge slat; $\alpha=15^{\circ}+10^{\circ} \sin \omega t, M_{\infty}=0.185$.

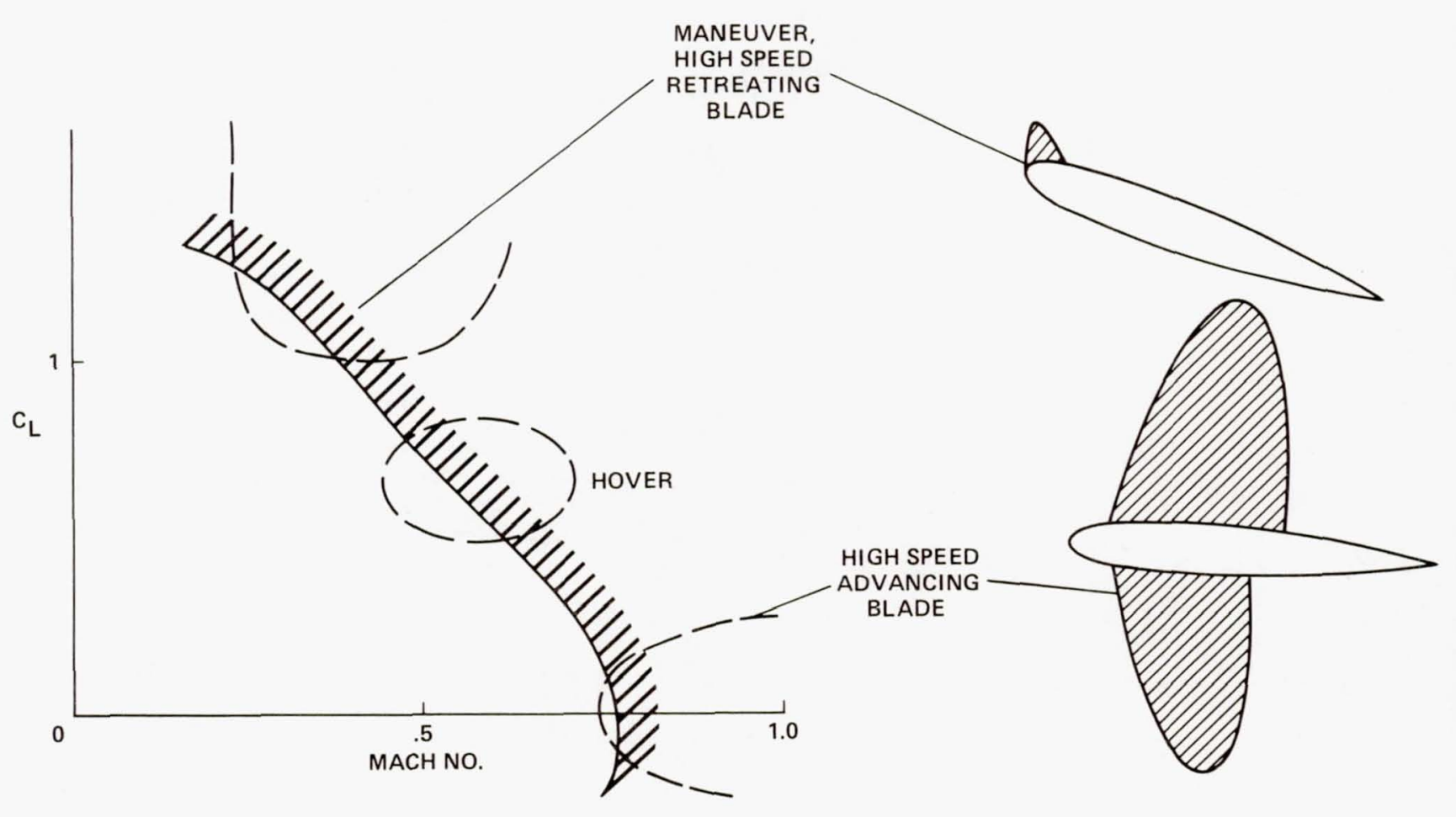

Figure 10.- Flow regimes for a rotor airfoil. 


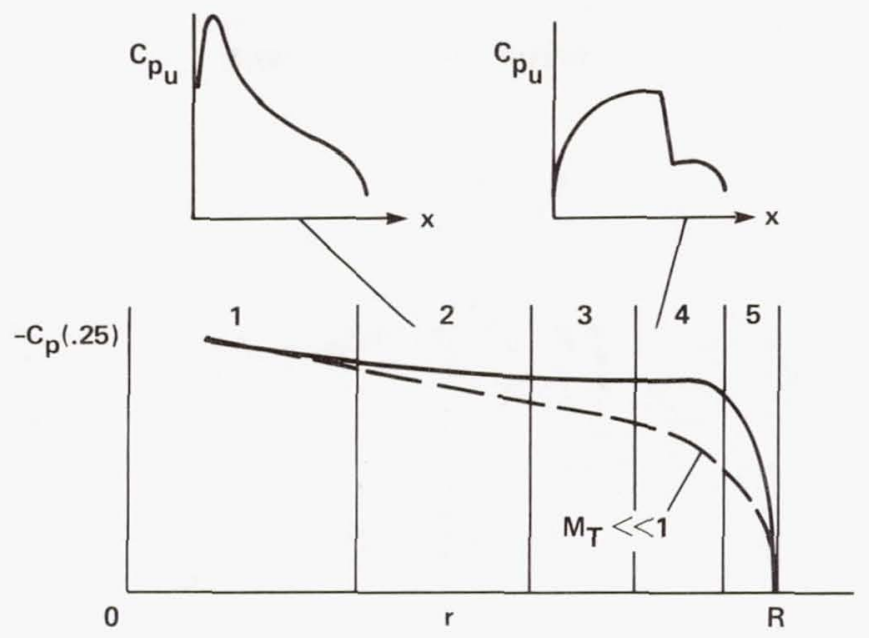

1: $K \rightarrow \infty$ INCOMPRESSIBLE

2: $K \gg 1$ COMPRESSIBLE, LINEAR, BLADE-ELEMENT

3: $K \simeq 1.5$ COMPRESSIBLE, NONLINEAR, NO SHOCKS

4: $K \sim 1$ TRANSONIC, NONLINEAR, SHOCKS

5: $\quad K \sim 1$ TRANSONIC, 3-D TIP RELIEF, WEAKER SHOCKS

Figure 11.- Spanwise variations and flow regimes for an advancing rotor blade.
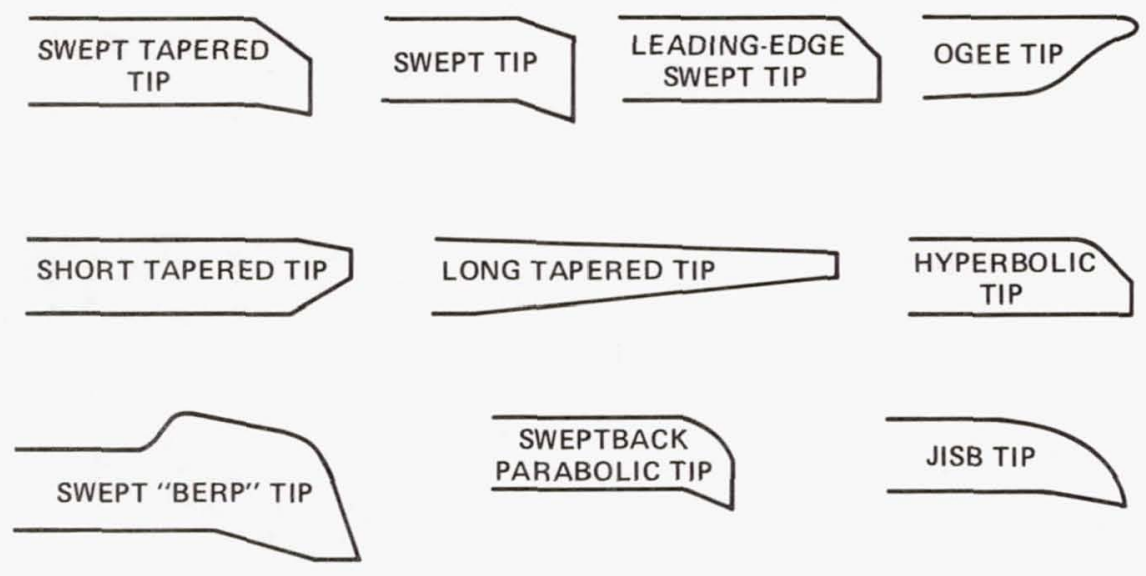

TIP

Figure 12.- Examples of advanced rotor blade tips. 

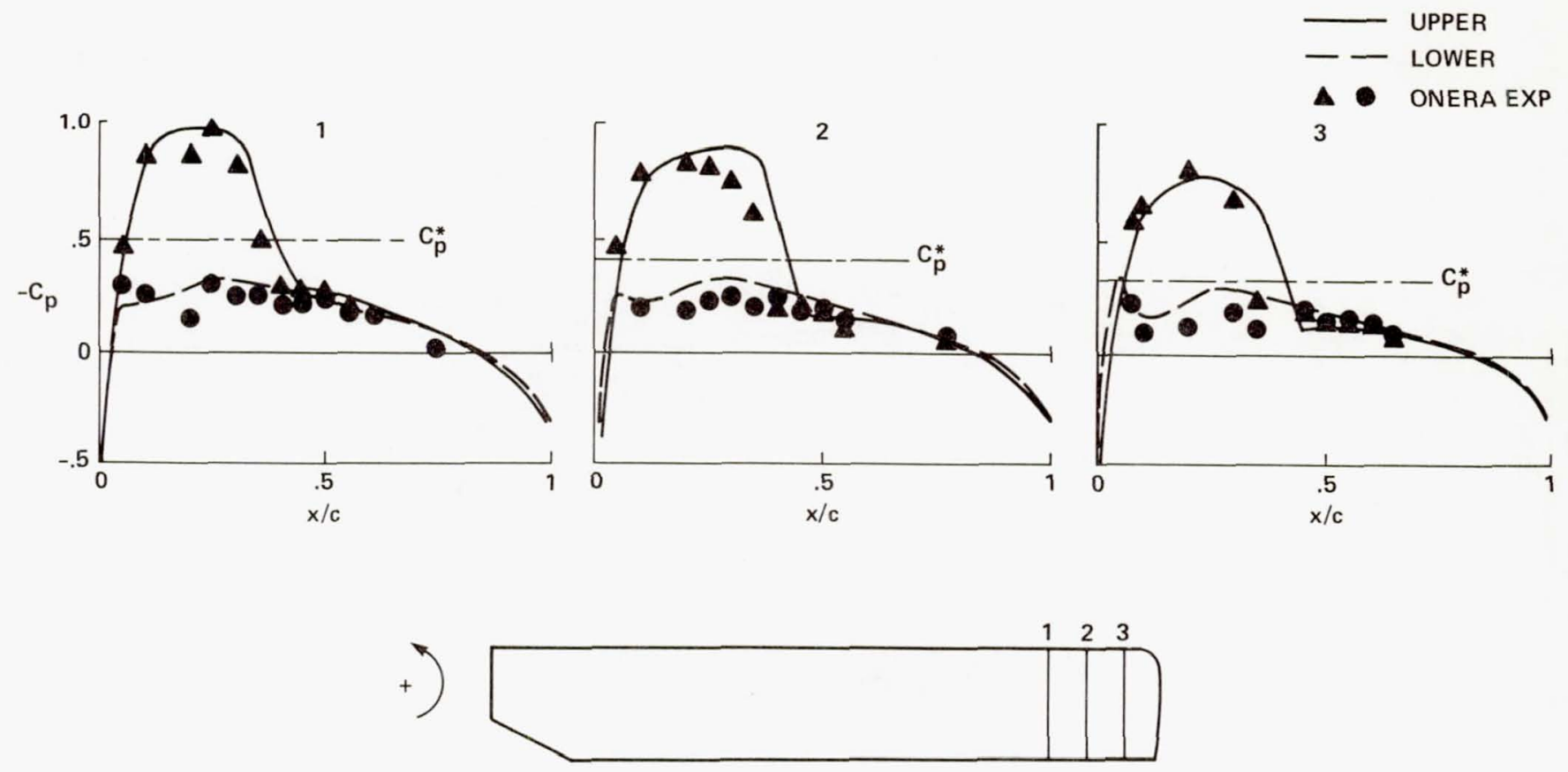

Figure 13.- Pressure distributions on a lifting rotor at high advance ratio, using an unsteady smal1-disturbance code (ref. 15); $\mu=0.36, \mathrm{C}_{\mathrm{T}} / \sigma=0.05, \psi=90^{\circ}$.

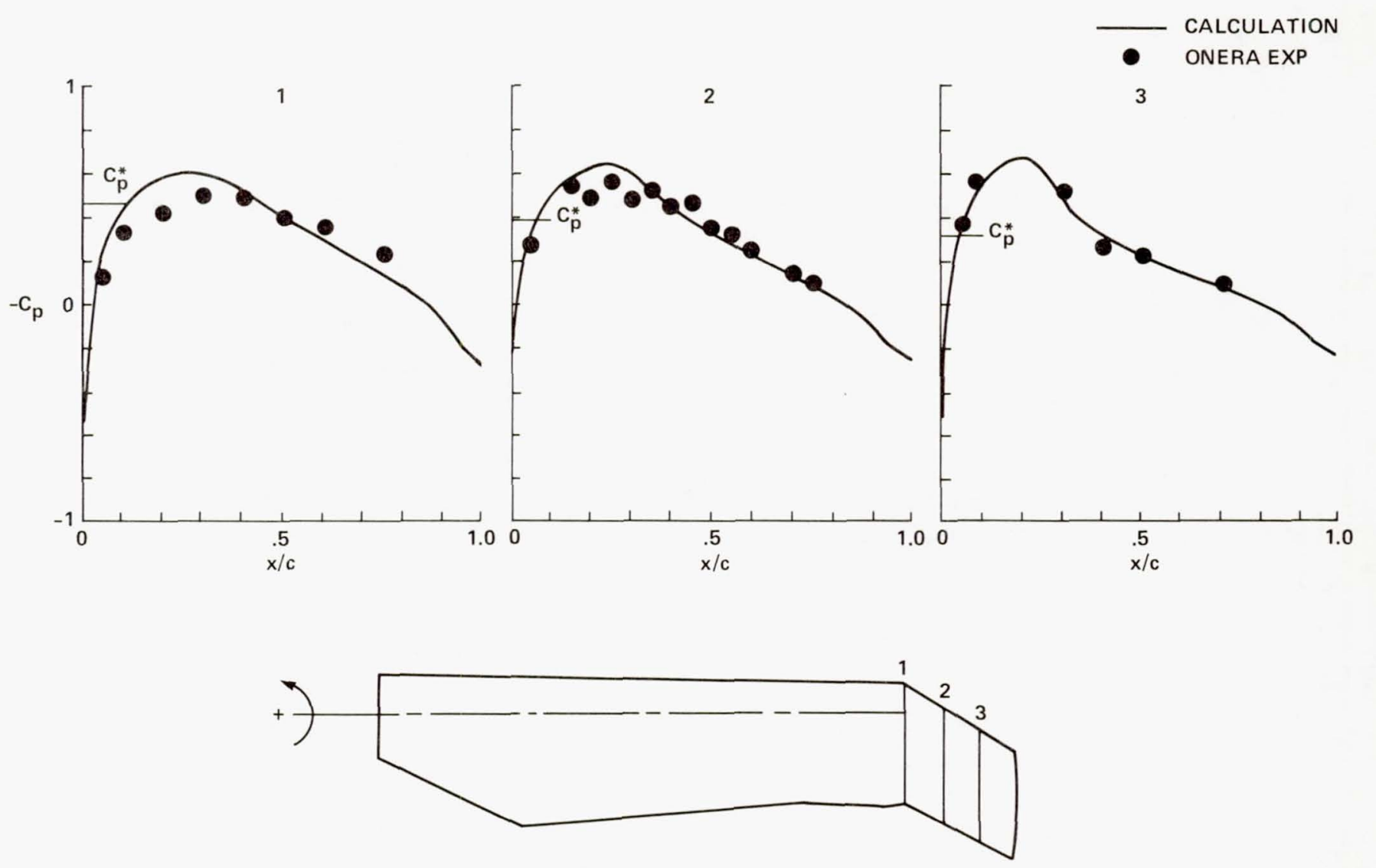

Figure 14.- Pressure distributions on a nonlifting rotor at high advance ratio, using a quasi-steady full-potential code (ref. 16); $\mu=0.40, \psi=90^{\circ}$. 


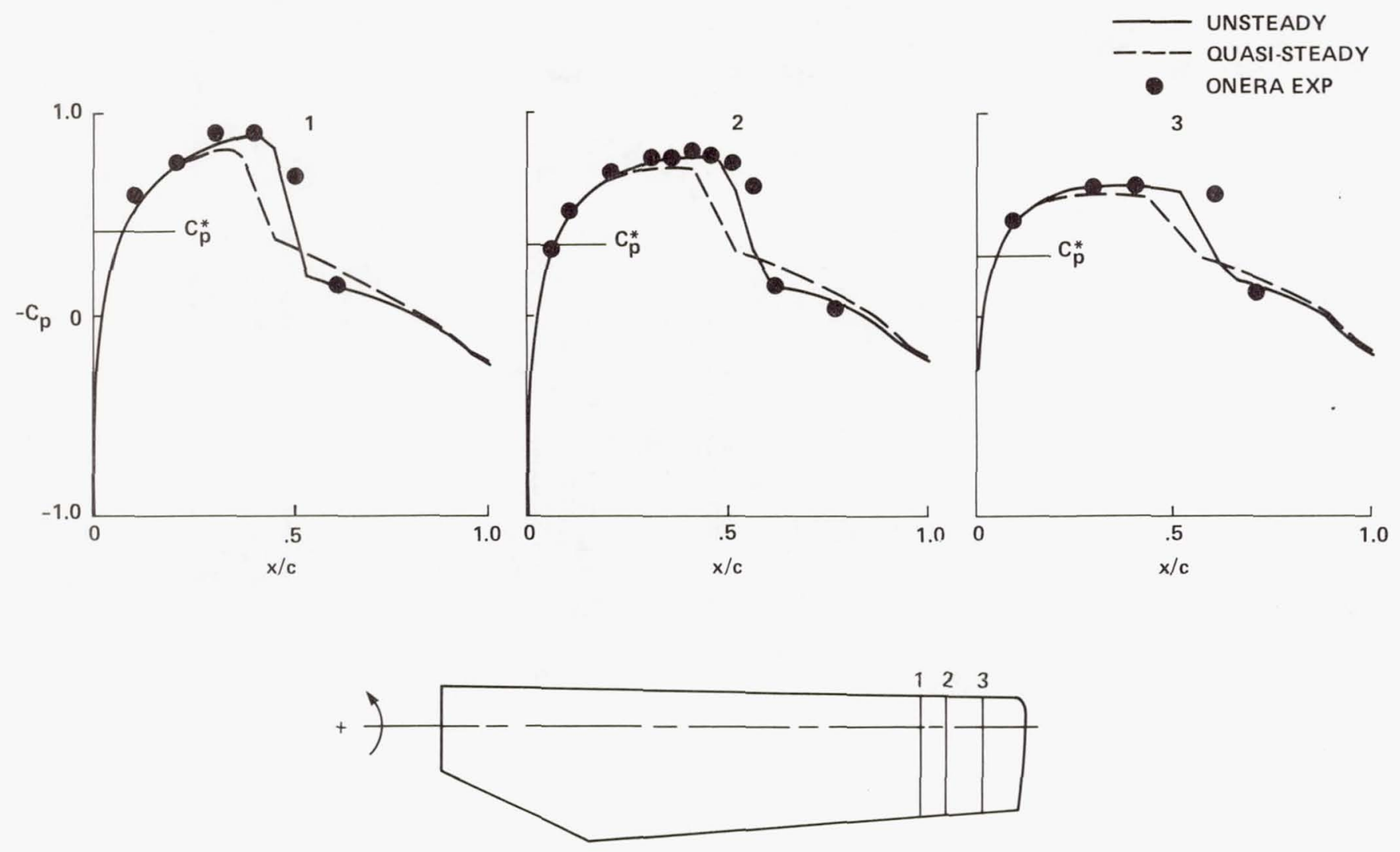

Figure 15.- Pressure distributions on a nonlifting rotor at high advance ratio, using an unsteady full-potential code; $\mu=0.55, \psi=120^{\circ}$.

MUST ACCOUNT FOR FLOW OUTSIDE OF MESH REGION

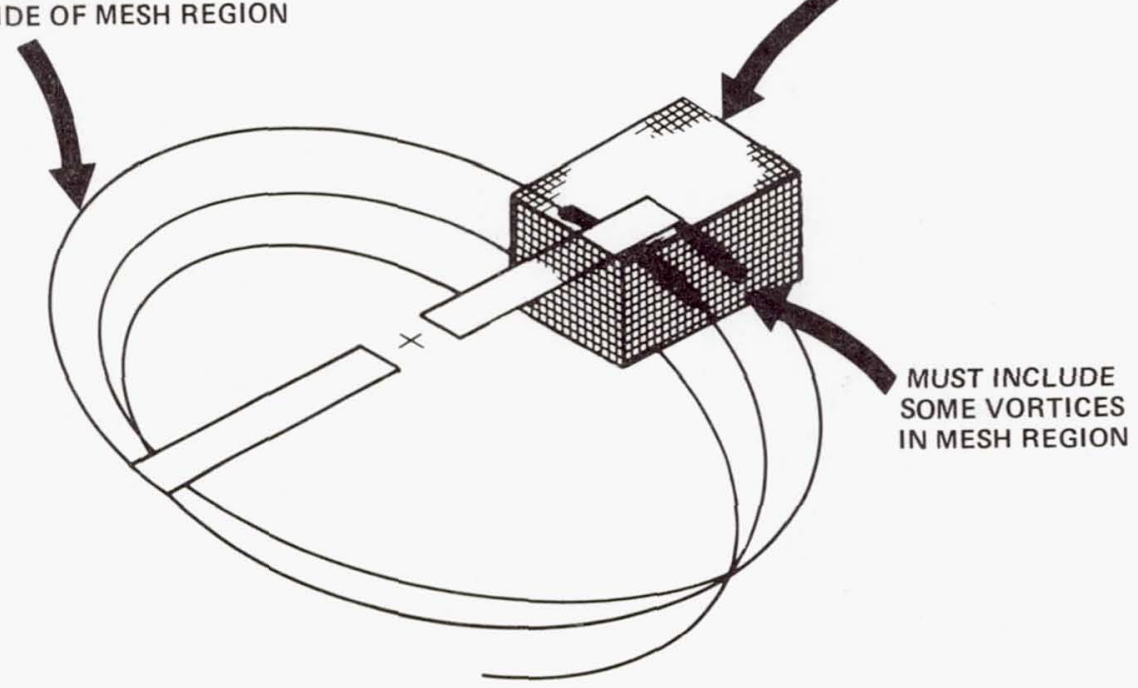

Figure 16.- Aerodynamic modeling of the rotor for finite-difference methods. 


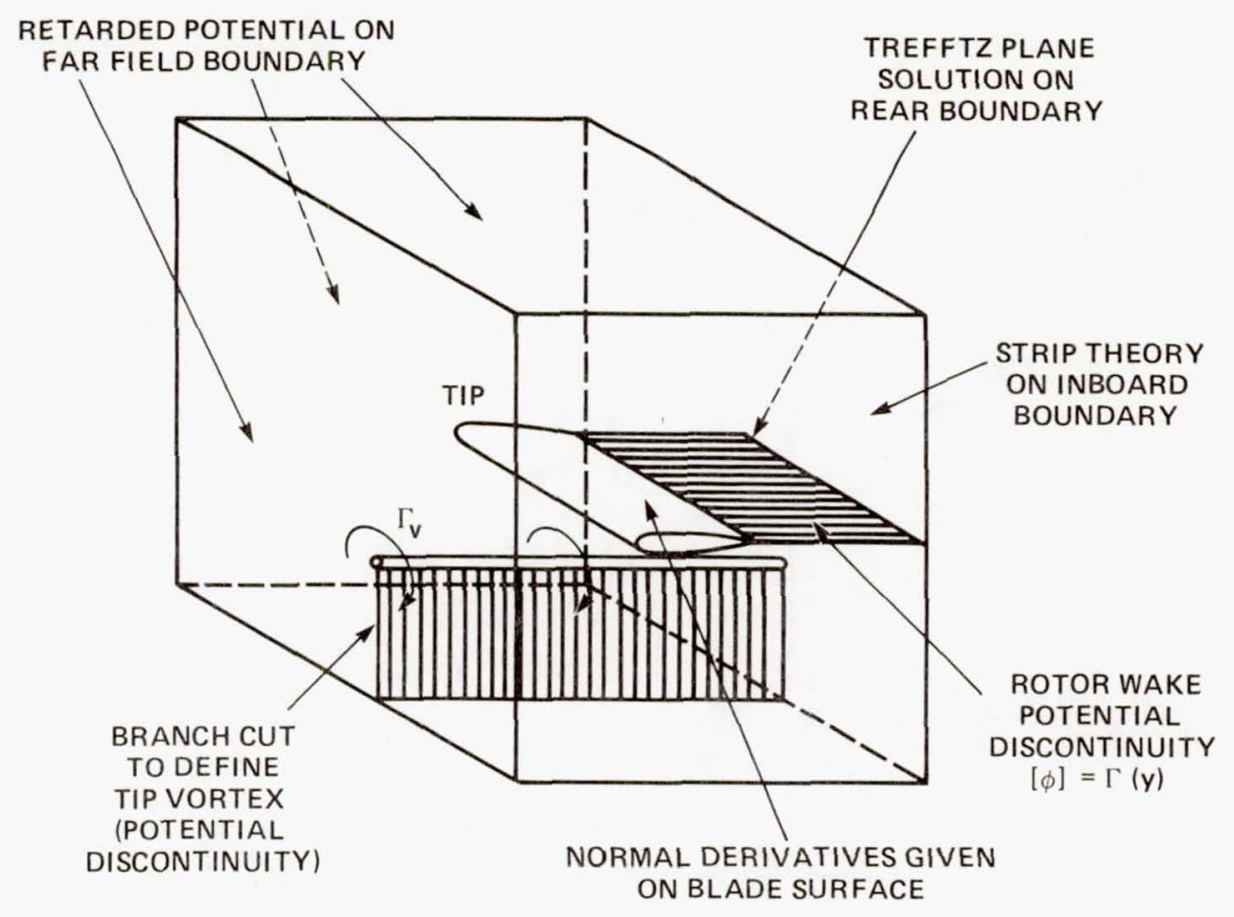

Figure 17.- Boundary conditions for potential flow past rotor blades.
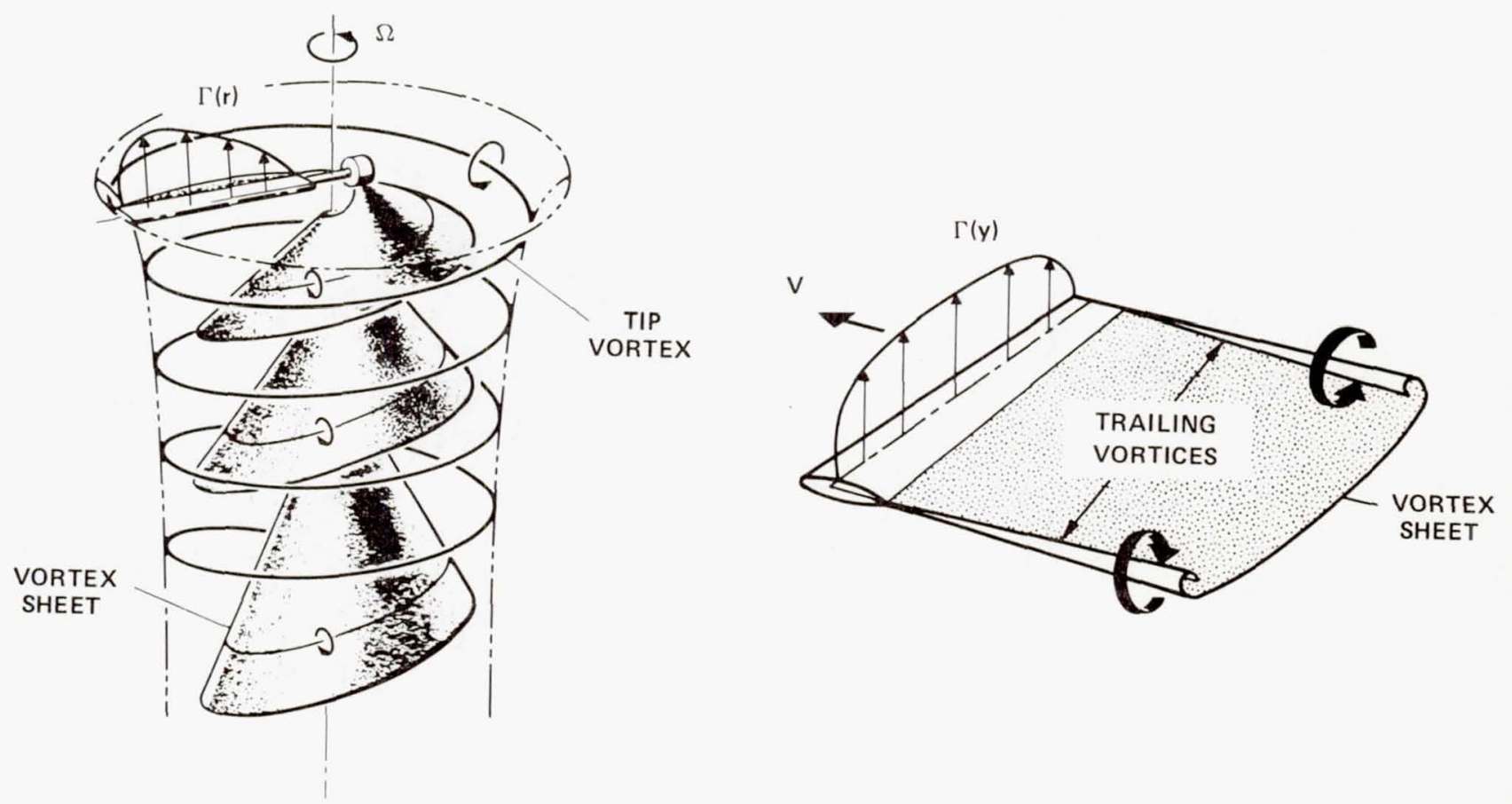

Figure 18.- Wake of a rotor in hover vs. a fixed wing wake. 

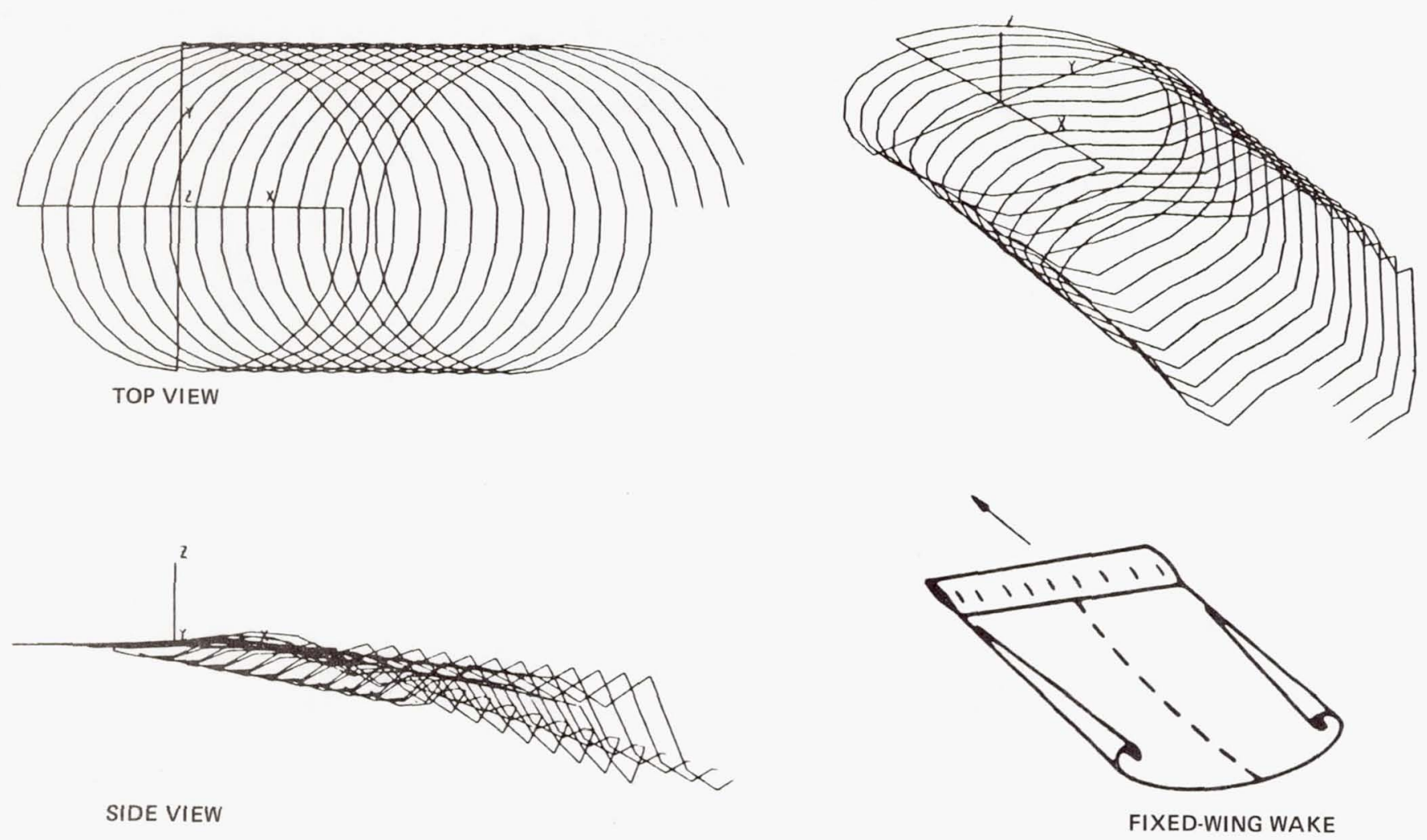

Figure 19.- Wake of a rotor in forward flight (ref. 17); 4 blades, $\mu=0.10$. 


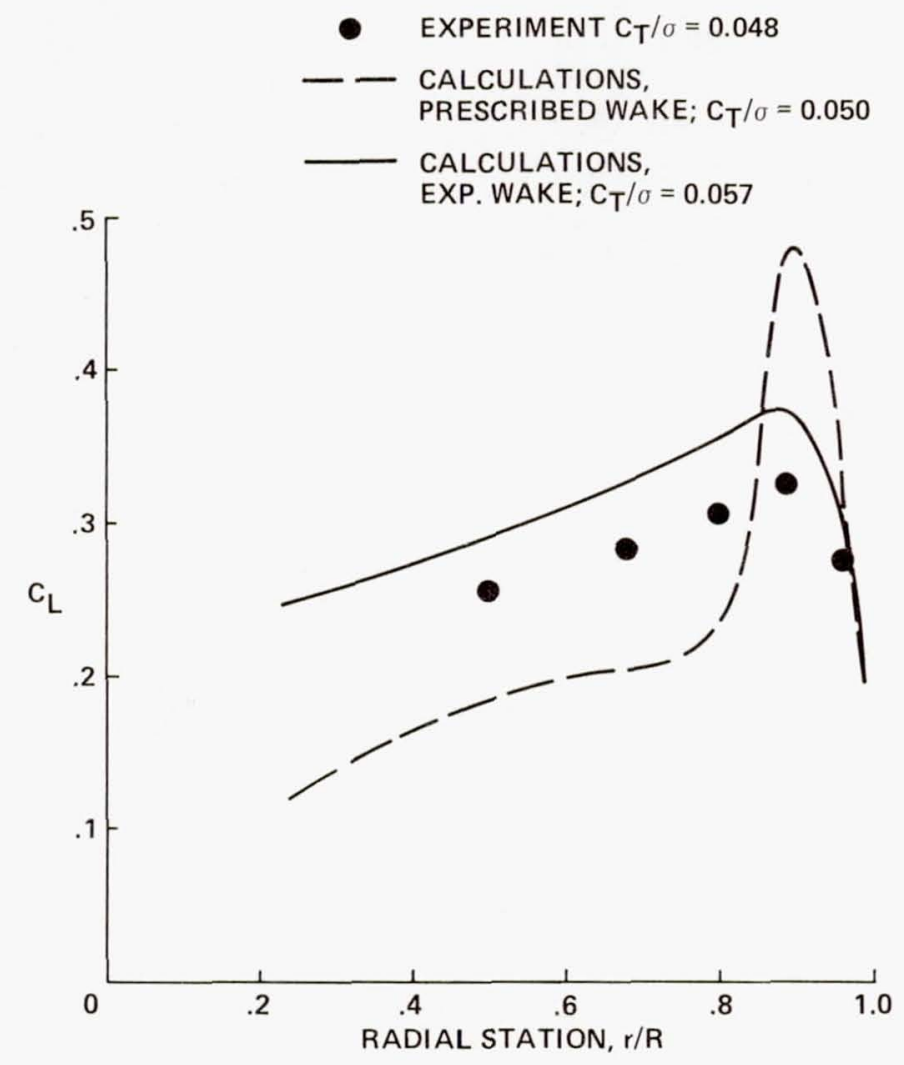

(a) Lifting-line calculations for prescribed and experimentally-derived wake geometries.

Figure 20.- Spanwise lift distribution in hover; $\mathrm{AR}=6, \theta_{C}=8^{\circ}$. 


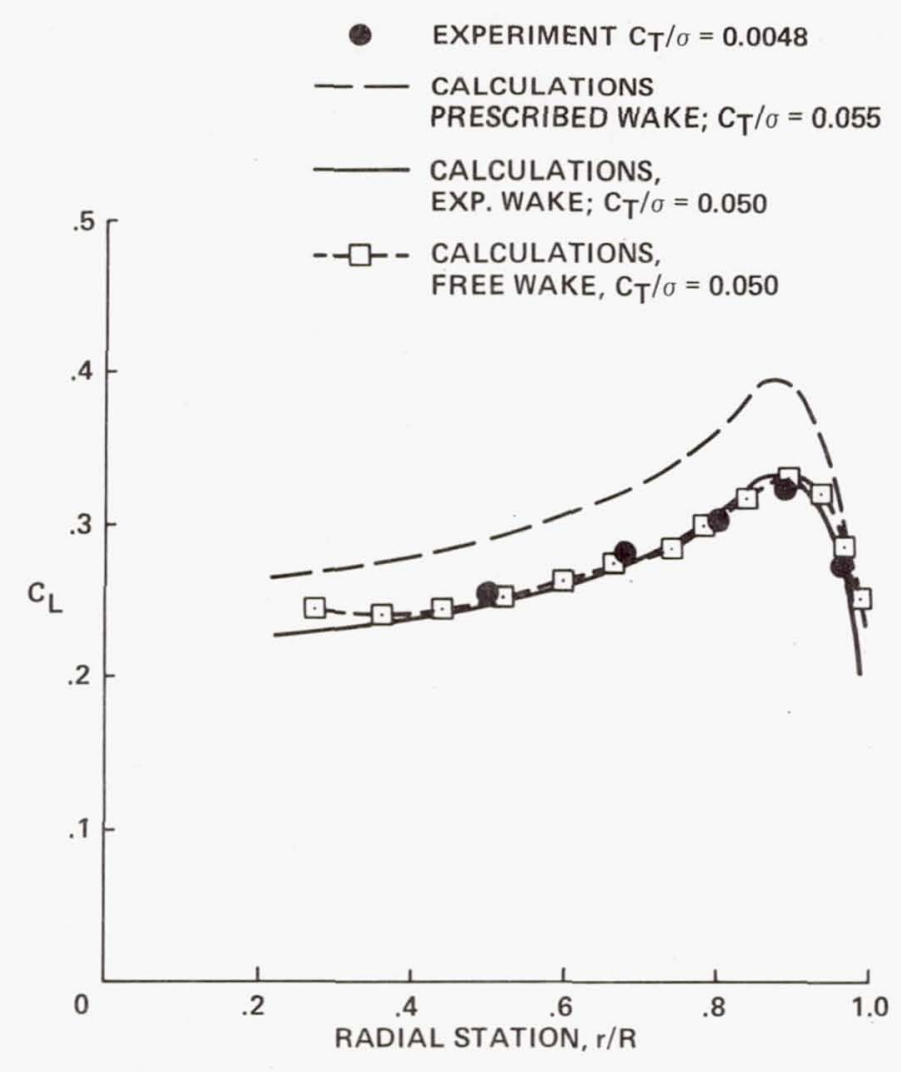

(b) Lifting-surface calculations for prescribed, free-wake and experimentallyderived wake geometries.

Figure 20.- Concluded. 


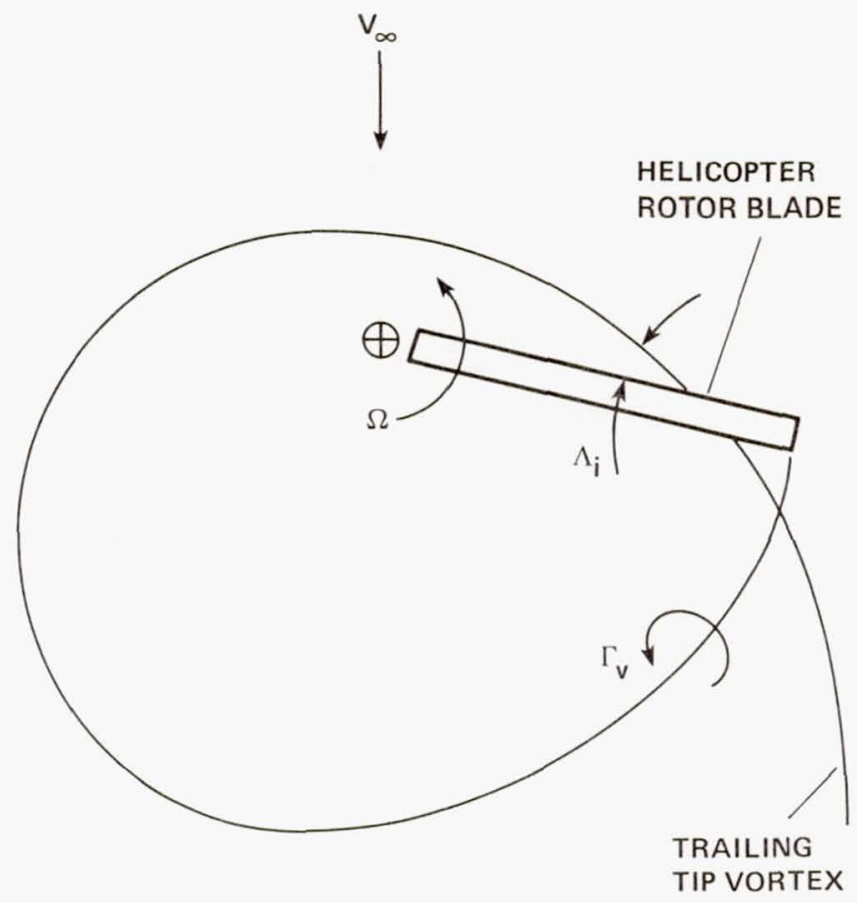

Figure 21.- Helicopter blade-vortex interaction.

IN GENERAL: 3-D, UNSTEADY

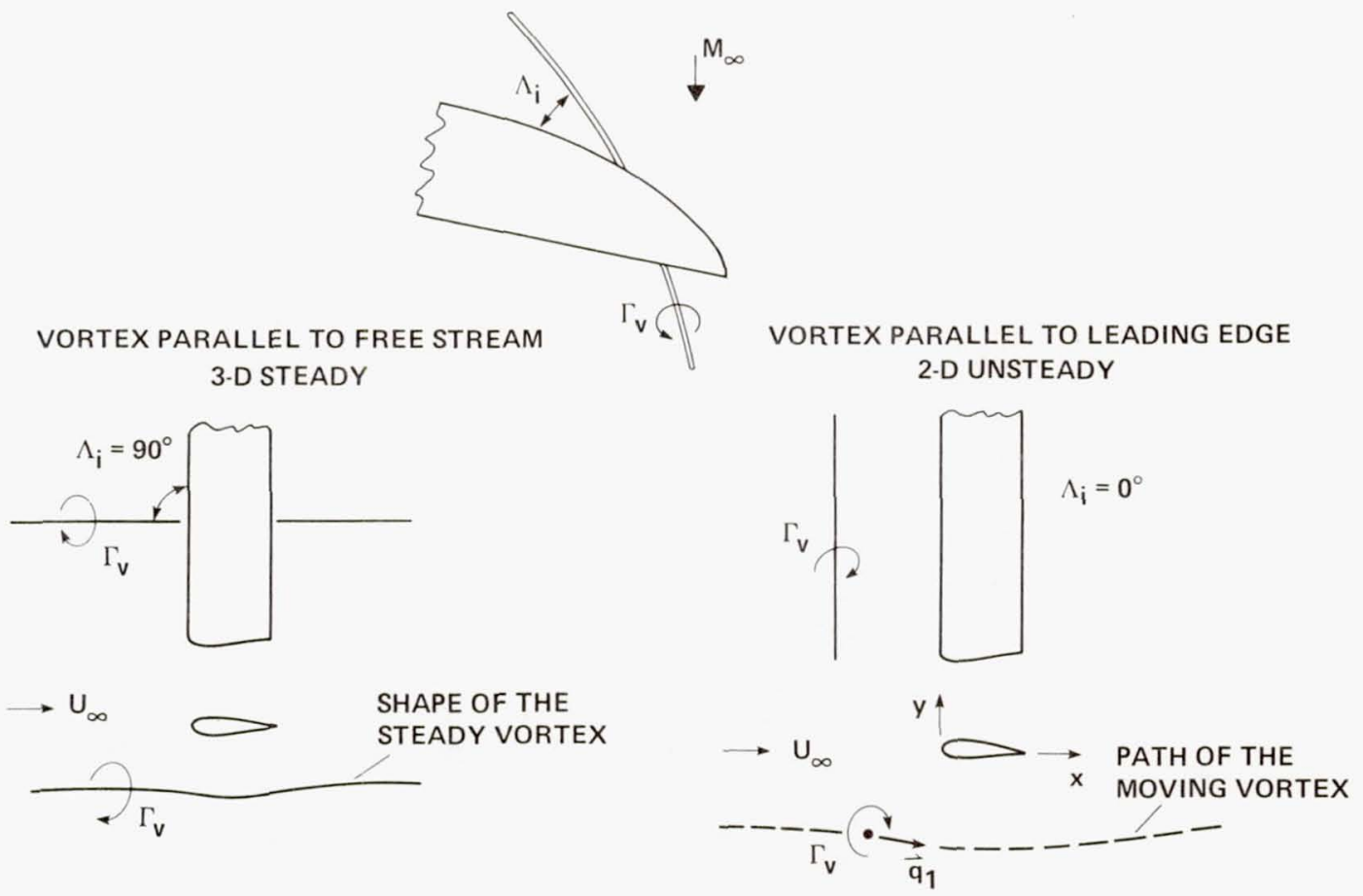

Figure 22.- Limiting cases of blade-vortex interaction. 


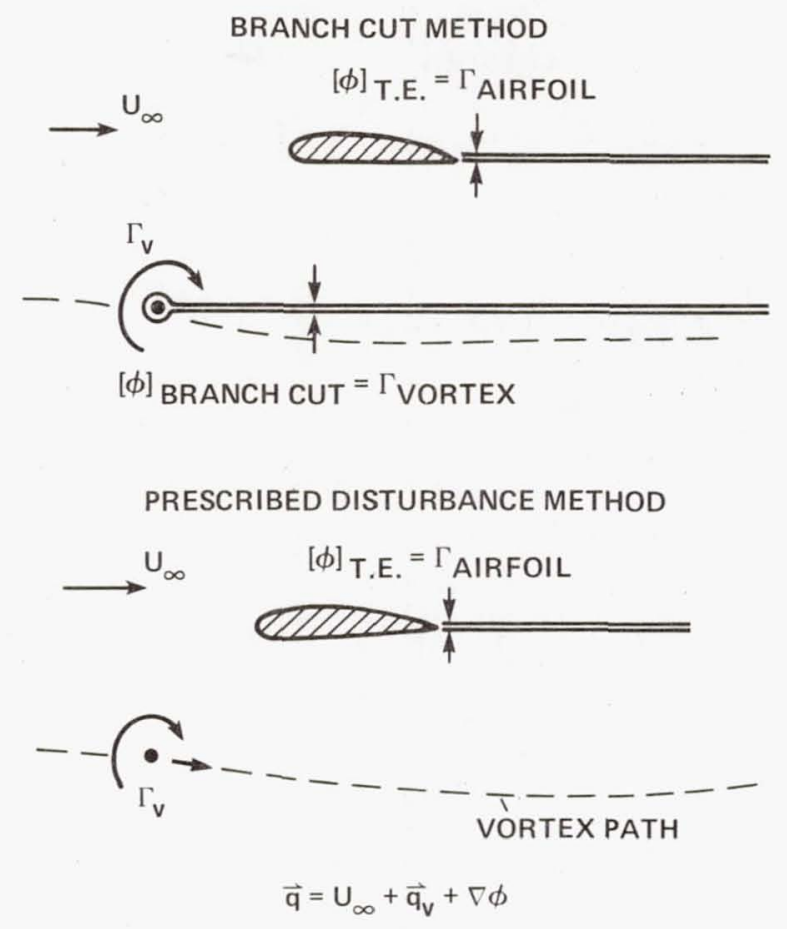

Figure 23.- Two methods of introducing a vortex into the flow field.
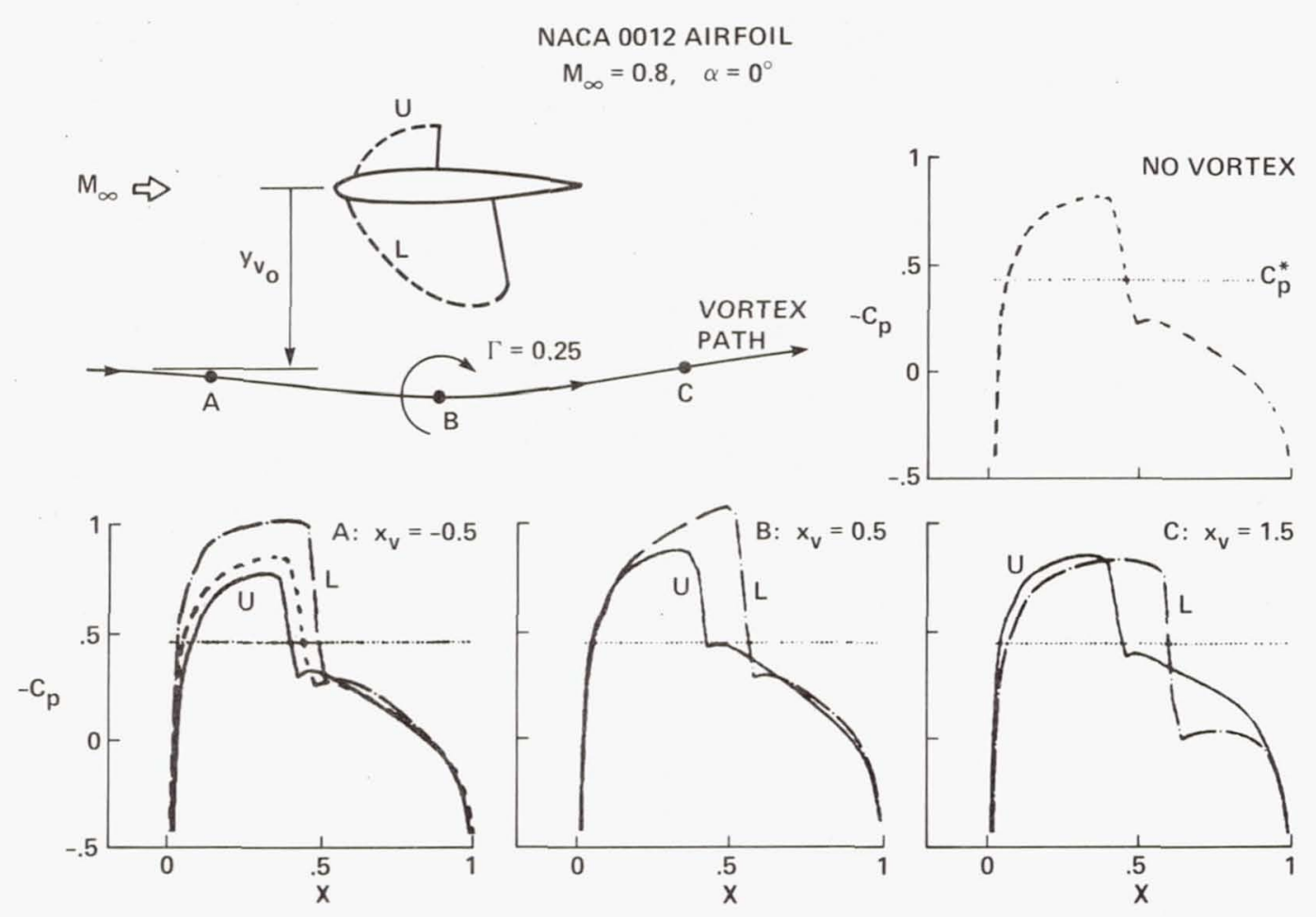

Figure 24.- Transonic airfoil-vortex interaction (ref. 23). 


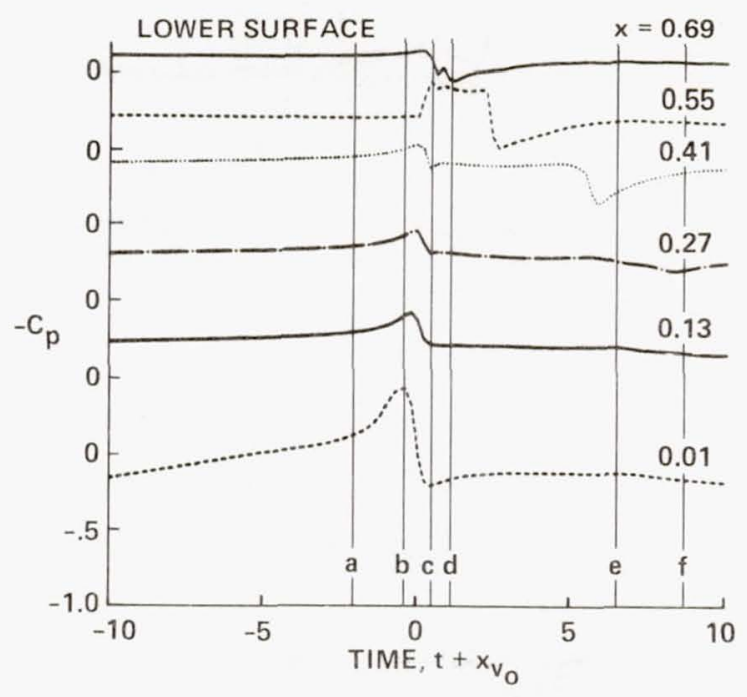

(a) Time histories at specific points on the airfoil.

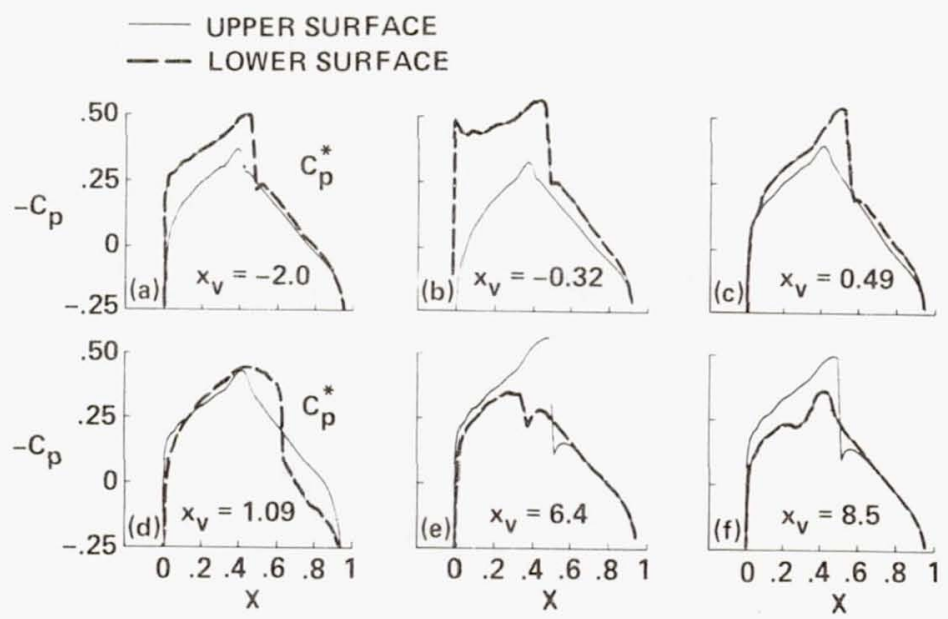

(b) Pressure distributions on the airfoil at specific times.

Figure 25.- Pressure variations on the lower surface during an airfoil-vortex interaction (ref. 23) NACA $64 \mathrm{A006}$ airfoil, $\mathrm{M}_{\infty}=0.85, \alpha=0, \Gamma=0.20$, $\mathrm{y}_{\mathrm{v}_{\mathrm{O}}}=-0.26$. 


\section{TILT-ROTOR DOWNLOADS}
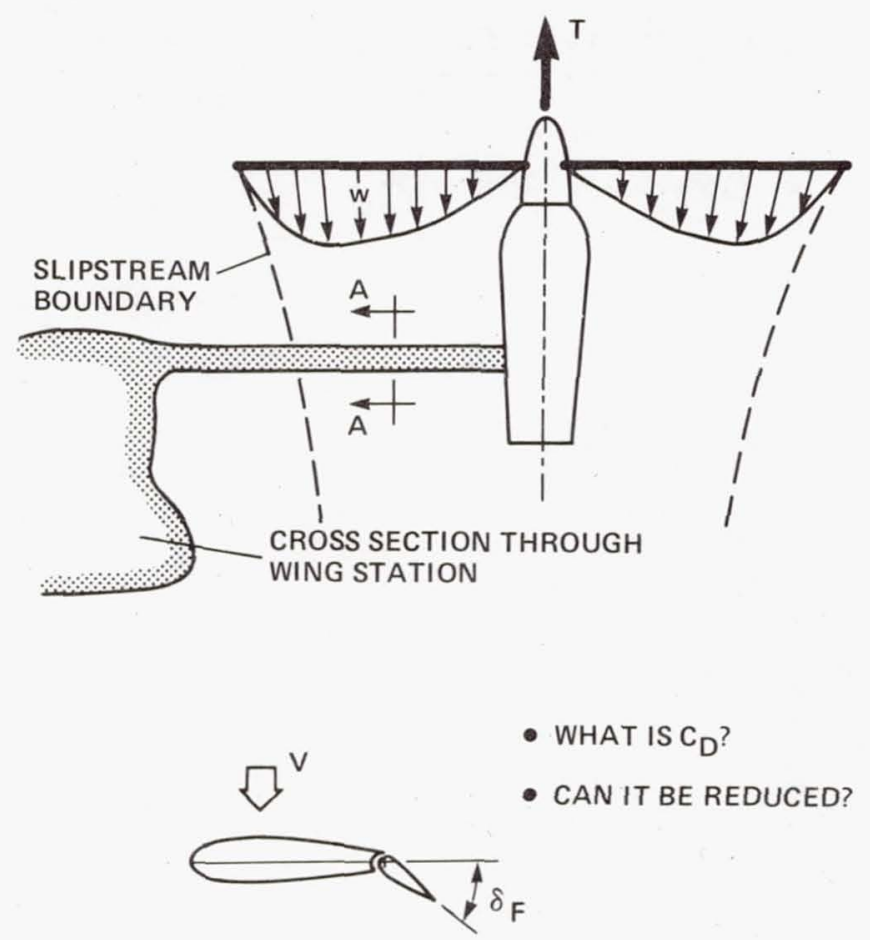

SECTION A-A

Figure 26.- The tilt-rotor aircraft in the hover mode. 


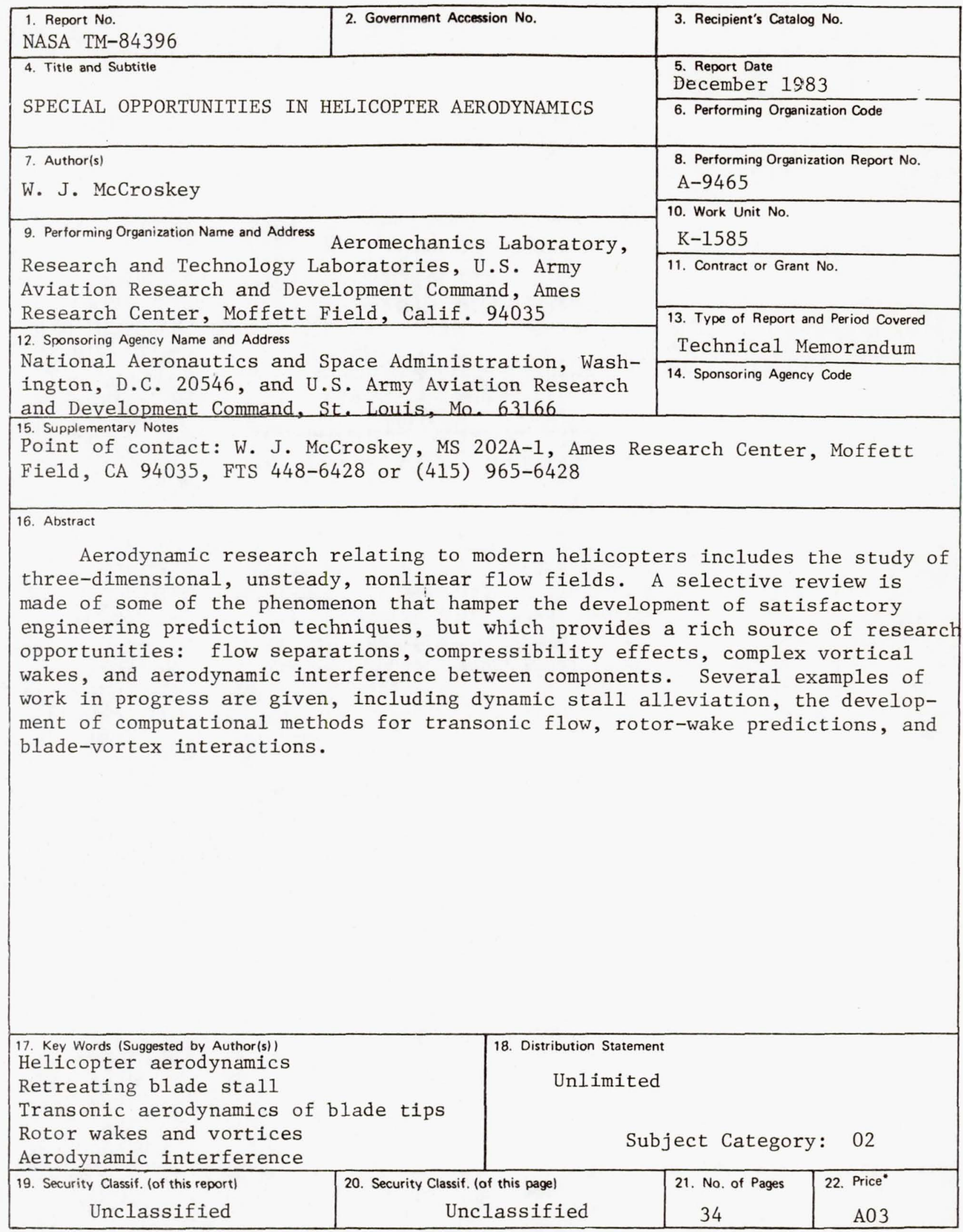

"For sale by the National Technical Information Service, Springfield, Virginia 22161 
